\title{
Comprehensive classification of the plant non-specific lipid transfer protein superfamily towards its Sequence - Structure - Function analysis
}

\author{
Cecile Fleury $^{1}$, Jerome Gracy ${ }^{2}$, Marie-Francoise Gautier ${ }^{1}$, Jean-Luc Pons ${ }^{2}$, Jean-Francois Dufayard ${ }^{3}$, Gilles \\ Labesse $^{2}$, Manuel Ruiz ${ }^{3}$, Frederic de Lamotte ${ }^{\text {Corresp. } 1}$ \\ 1 UMR AGAP, INRA, Montpellier, France \\ 2 CBS, CNRS Univ Montpellier INSERM, Montpellier, France \\ 3 UMR AGAP, CIRAD, Montpellier, France \\ Corresponding Author: Frederic de Lamotte \\ Email address: frederic.de-lamotte@inra.fr
}

Background. Non-specific Lipid Transfer Proteins (nsLTPs) are widely distributed in the plant kingdom and constitute a superfamily of related proteins. Several hundreds of different nSLTP sequences - and counting - have been characterized so far, but their biological functions remain unclear. It has been clear for years that they present a certain interest for agronomic and nutritional issues. Deciphering their functions means collecting and analyzing a variety of data from gene sequence to protein structure, from cellular localization to the physiological role. As a huge and growing number of new protein sequences are available nowadays, extracting meaningful knowledge from sequence-structure-function relationships calls for the development of new tools and approaches. As nsLTPs show high evolutionary divergence, but a conserved common right handed superhelix structural fold, and as they are involved in a large number of key roles in plant development and defense, they are a stimulating case study for validating such an approach.

Methods. In this study we comprehensively investigated 797 nsLTP protein sequences, including a phylogenetic analysis on canonical protein sequences, three-dimensional (3D) structure modelling and functional annotation using several well-established bioinformatics programs. Additionally, two integrative methodologies using original tools were developed. The first was a new method for the detection of i) conserved amino acid residues involved in structure stabilization and ii) residues potentially involved in ligand interaction. The second was a structure-function classification based on the Evolutionary Trace Display method using a new tree visualization interface. We also present a new tool for visualizing phylogenetic trees.

Results. Following this new protocol, an updated classification of the nSLTP superfamily was established and a new functional hypothesis for key residues is suggested. Lastly, this work allows a better representation of the diversity of plant nsLTPs in terms of sequence, structure and function. 
1 Comprehensive classification of the plant non-specific

2 lipid transfer protein superfamily towards its

3 Sequence - Structure - Function analysis

Cécile Fleury ${ }^{1}$, Jérôme Gracy ${ }^{3}$, Marie-Françoise Gautier ${ }^{1}$, Jean-Luc Pons ${ }^{3}$, Jean-François

6 Dufayard $^{2}$, Gilles Labesse ${ }^{3}$, Manuel Ruiz ${ }^{2}$, Frédéric de Lamotte ${ }^{1}$

${ }^{1}$ INRA, UMR AGAP, F-34060 Montpellier, France

${ }^{2}$ CIRAD, UMR AGAP, F-34398, Montpellier

${ }^{3}$ CBS CNRS Univ. Montpellier- INSERM, Montpellier, F-34090, France

Corresponding Author:

Frédéric de Lamotte ${ }^{1}$

Avenue Agropolis - TA A-108/03 - Montpellier - F-34398 Cedex 5 - France

Email address: frederic.de-lamotte@inra.fr

\section{ABSTRACT}

Background. Non-specific Lipid Transfer Proteins (nsLTPs) are widely distributed in the plant kingdom and constitute a superfamily of related proteins. Several hundreds of different nsLTP sequences - and counting - have been characterized so far, but their biological functions remain unclear. It has been clear for years that they present a certain interest for agronomic and nutritional issues. Deciphering their functions means collecting and analyzing a variety of data from gene sequence to protein structure, from cellular localization to the physiological role. As a huge and growing number of new protein sequences are available nowadays, extracting meaningful knowledge from sequence-structure-function relationships calls for the development of new tools and approaches.

As nsLTPs show high evolutionary divergence, but a conserved common right handed superhelix structural fold, and as they are involved in a large number of key roles in plant development and defense, they are a stimulating case study for validating such an approach. Methods. In this study we comprehensively investigated 797 nsLTP protein sequences, including a phylogenetic analysis on canonical protein sequences, three-dimensional (3D) structure modelling and functional annotation using several well-established bioinformatics programs. Additionally, two integrative methodologies using original tools were developed. The first was a new method for the detection of i) conserved amino acid residues involved in structure stabilization and ii) residues potentially involved in ligand interaction. The second was a structure-function classification based on the Evolutionary Trace Display method using a new tree visualization interface. We also present a new tool for visualizing phylogenetic trees. Results. Following this new protocol, an updated classification of the nsLTP superfamily was established and a new functional hypothesis for key residues is suggested. 
40 Lastly, this work allows a better representation of the diversity of plant nsLTPs in terms of

41 sequence, structure and function.

42

\section{INTRODUCTION}

Since the work of Kader (Kader et al., 1984; Kader, 1996), numerous proteins capable of transferring lipids have been annotated as non-specific lipid transfer proteins (nsLTPs). Their primary sequences are characterized by a conserved 8-Cysteine Motif (8CM) (C-Xn-C-Xn-CC$\mathrm{Xn}-\mathrm{CXC}-\mathrm{Xn}-\mathrm{C}-\mathrm{Xn}-\mathrm{C}$ ), which plays an important role in their structural scaffold (José-Estanyol et al., 2004). Based on their molecular masses, plant nsLTPs were first separated into two types: type I $(9 \mathrm{kDa})$ and type II $(7 \mathrm{kDa})$, which were distinct both in terms of primary sequence identity and the disulfide bond pattern (Douliez et al., 2001).

Plant nsLTPs are ubiquitous proteins encoded by multigene families, as reported in different phylogenetic studies. However, these studies involve a limited number of sequences and/or species: fifteen nsLTPs identified in Arabidopsis (Arondel et al., 2000), restricted to Poaceae (Jang et al., 2007), Solanaceae (Liu et al., 2010, D’Agostino et al., 2019), or Gossypium (Li et al., 2016; Meng et al., 2018). Recently 189 nsLtp genes were identified in 3 Gossypium species (Li et al., 2016) and 138 nsLtp genes in the single Gossypium hirsutum species (Meng et al., 2018) using traditional sequence homology approaches. As for Solanum lycopersicum, D'Agostino and collaborators identified 64 nsLtp gene sequences using an Hidden Markov Model approach (D’Agostino et al., 2019). Around 200 nsLTPs have been identified in wheat, rice and Arabidopsis genomes and classified into nine different types according to sequence similarity (Boutrot et al., 2008). More extensive studies including ancestral plants indicate that nsLTPs are also present in liverworts, mosses and ferns, but not present in algae (Edstam et al., 2011; Wang et al., 2012). However, the efforts made so far to classify the members of the nsLTP superfamily were including proteins that do not satisfy the strict criteria of 8CM pattern (Edstam et al., 2011; Wang et al., 2012). In comparison to previous studies, we computed the most extensive phylogenetic analysis, sampling 797 nsLTP sequences from 123 different species. This allows to enrich the phylogenic tree of many evolutionary events that would have been hidden with more restrictive species choices. These events are essentially gene duplications and have a major influence on the family evolution that could be correlated to 3D structure evolution. From a structural point of view, the nsLTP family belongs to the all-alpha class in the SCOP database (Murzin et al., 1995), as these small proteins contain four or five helices organized in a right-handed superhelix. To date, only 30 three-dimensional redundant structures corresponding to 8 different proteins have been experimentally determined. According to SCOP, the protein fold called "Bifunctional inhibitor/lipid-transfer protein/seed storage $2 \mathrm{~S}$ albumin" is found in at least six distinct plant nsLTPs for which the 3D structure has been solved (from five species Triticum aestivum, Hordeum vulgare, Zea mays, Oryza sativa and Triticum turgidum), and one soybean hydrophobic protein. In the RCSB Protein Database (Berman et al., 2000) we listed four more plant nsLTP 3D structures (from Nicotiana tabacum, Phaseolus aureus, Prunus persica and Arabidopsis thaliana). According to the CATH database (Orengo et al., 1997), nsLTPs 
80 belong to the "Mainly alpha" class. They display the "Orthogonal Bundle" architecture and the 81 "Hydrophobic Seed Protein" topology. At this level, only one homologous superfamily called 82 "Plant lipid-transfer and hydrophobic proteins" can be found. The superfamily appears to contain 83 ten distinct protein sequences, lacking the $A$. thaliana nsLTP, but including the soybean 84 hydrophobic protein found in the SCOP database. Of the known nsLTP 3D structures, only 85 Boutrot's type I, II and IV are represented. An interesting point to be noted is that two different 86 cysteine pairing patterns have been observed (which correspond to a single cysteine switch 87 between two disulfide bridges): C1-C6 and C5-C8 in type I structures; C1-C5 and C6-C8 in type 88 II and IV structures. However, C2-C7 and C3-C4 bridges are common to all known nsLTP 89 structures and the overall fold is conserved among the whole family.

90 From a functional point of view, plant nsLTPs are classified into different families depending on 91 the scope of interest and their properties (Liu et al. 2015). Plant nsLTPs belong to the Prolamin superfamily (AF050), which includes the largest number of allergens (Radauer et al., 2008). Indeed, several nsLTPs from fruits of the Rosaceae family, nuts or different vegetables are food allergens, with fruit nsLTPs being mainly localized in the peel (Salcedo et al., 2007, D'Agostino et al., 2019).

Plant nsLTPs are members of the pathogenesis-related proteins and compose the PR14 family (van Loon et al., 2006). Their role in plant defense mechanisms has been shown by the induction of nsLtp gene expression following pathogen infections, overexpression in transgenic plants, or their antimicrobial properties (Molina \& García-Olmedo, 1993; Cammue et al., 1995; Li et al., 2003; Girault et al., 2008; Sun et al., 2008). A role in plant defense signaling pathways has also been suggested for an Arabidopsis type IV nsLTP, which needs to form a complex with glycerol3-phosphate for its translocation and induction of systemic acquired resistance (Maldonado et al., 2002; Chanda et al., 2011). One wheat nsLTP competes with a fungal cryptogein receptor in tobacco plasma membranes and, when the LTP is complexed with lipids, its interaction with the membrane and its defense activity are enhanced (Buhot et al., 2001; Buhot et al., 2004). In wheat, nsLtp genes display a complex expression pattern during the development of the seed (Boutrot et al., 2005). NsLTPs may also be involved in plant defense mechanisms through their participation in cuticle synthesis (Debono et al., 2009). This function is supported by their extracellular localization (Thoma et al., 1993; Pyee et al., 1994), the expression of different

111 1999), a positive correlation between nsLtp gene expression and cuticular wax deposition

112 (Cameron et al., 2006), and their ability to bind cutin monomers (i.e. hydroxylated fatty acids)

113 (Douliez et al., 2001). In addition, nsLtp gene transcripts are abundant or specifically present in 114 trichomes and one tobacco nsLTP seems to be required for lipid secretion from glandular 115 trichomes indicating that nsLTPs may play a role either in the secretion of essential oils or in 116 defense mechanism (Lange et al., 2000; Aziz et al., 2005; Choi et al., 2012). Several nsLtp 117 genes are up or down-regulated by application of different abiotic stresses including low 118 temperature, drought, salinity and wounding (Wang et al., 2012; Treviño \& O'Connell, 1998; 119 Gaudet et al., 2003; Maghuly et al., 2009). A cabbage nsLTP isolated from leaves stabilizes 
120 thylakoid membranes during freezing (Sror et al., 2003). Transgenic orchids transformed with a

121 rice nsLTP exhibit an enhanced tolerance to cold stress (Qin et al., 2011).

122 Function in male reproductive tissues has also been shown for a lily nsLTP involved in pollen

123 tube adhesion (Mollet et al., 2000; Park et al., 2000) and the Arabidopsis LTP5 implicated in

124 pollen tube guidance in the pistil (Chae et al., 2009; Chae \& Lord, 2011). A tobacco nsLTP that

125 accumulates in pistils has been shown to be involved in cell wall loosening, and this activity

126 relies on the hydrophobic cavity of the protein (Nieuwland et al., 2005).

127 Non-specific LTPs are possibly involved in a range of other biological processes, but their

128 physiological functions are not clearly understood. An analysis of gain of function or defective

129 plant mutants can address these issues (Maldonado et al., 2002; Chae et al., 2009). Site directed

130 mutagenesis (SDM) led to the identification of residues involved in their antifungal activity, lipid

131 binding and lipid transfer (Ge et al., 2003; Cheng et al., 2008; Sawano et al., 2008). However,

132 even if they remain extremely valuable, these approaches are time-consuming and have so far

133 been limited to a small number of proteins. They need to be computationally assisted, using

134 information emerging from big datasets.

135 Current bioinformatics programs such as GeneSilico Metaserver (Kurowski \& Bujnicki, 2003) or

136 MESSA (Cong \& Grishin, 2012) provide an overview of known information about protein

137 sequences, structures and functions. However, studying inner relationships into such complex

138 superfamilies of proteins as the nsLTP superfamily requires a knowledge visualization and

139 classification tool that still needs to be developed.

140 This paper describes both the development of new tools together with the use of these tools to

141 improve our comprehension of the nsLTP superfamily.

142

143

144

145

\section{MATERIALS \& METHODS}

\section{1/ NsLTP sequences}

146

147

\section{Definition of the protein sequence set}

A first pool of plant nsLTPs sequences was retrieved from the UniProtKB (Swiss-Prot + TrEMBL) (http://www.uniprot.org), Phytozome (http://www.phytozome.net) and NCBI databases (http://www.ncbi.nlm.nih.gov), using either Blast or keyword queries ("Plant lipid transfer protein", "viridiplantae lipid transfer protein", "plant A9 protein", "A9 like protein", "tapetum specific protein", "tapetum specific", "anther specific protein", "A9 Fill”). Original data obtained on the Theobroma cacao genome were also investigated (Argout et al., 2011). From this large pool of proteins, the plant nsLTP dataset was defined according to a new set of criteria: (i) sequences from 60 to 150 residues long, including signal peptide; (ii) containing strictly eight cysteine residues after removal of the signal peptide; (iii) cysteine residues distributed in the 8CM pattern (C-Xn-C-Xn-CC-Xn-CXC-Xn-C-Xn-C). We excluded multidomain proteins, i.e. the hybrid proline-rich and hybrid glycine-rich proteins in which the signal peptide is followed by a proline-rich or a glycine-rich domain of variable length (José-Estanyol

160 et al., 2004). For each sequence, the signal peptide was detected and removed using SignalP 3.0 (Bendtsen et al., 2004). In all, including the wheat, rice and Arabidopsis sequences previously 
162 identified by Boutrot (Boutrot et al., 2008), 797 non-redundant mature amino acid sequences

163 belonging to more than 120 plant species were kept for analysis. This dataset is available online

164 (doi:10.18167/DVN1/UNKLA6).

165

166

167

\section{Sequence alignments and phylogenetic analysis}

In order to achieve the best alignment, the pool of 797 sequences was aligned using both the

169 MAFFT (Katoh et al., 2002; Katoh \& Toh, 2010) and MUSCLE (Edgar, 2004) programs with

170 respective parameters of 1.53 for gap opening, 0.123 for gap extension and BLOSUM62 matrix; maximum iteration 16 .

172 The two resulting multiple alignments were compared and conflicts between the two were highlighted. To discriminate between the two different cysteine patterns suggested (see Results section), a restricted analysis was carried out using only the $10 \mathrm{nsLTPs}$ for which at least one structure had previously been experimentally determined. Two new 10 -sequence alignments were calculated, one by MUSCLE and one by MAFFT. Using the ViTo program (Catherinot \& Labesse, 2004), each alignment was projected on type I, II and IV nsLTP 3D structures, and the spatial distance of equivalent cysteine residues was evaluated. The alignment that minimized these distances was selected as the best one. Based on the best alignment, a phylogenetic tree was calculated using PhyML (Guindon et al., 2010). Lastly, the tree was reconciled with the overall species tree using the Rap-Green program (Dufayard et al., 2005).

184

\section{2/ NsLTP three-dimensional structures}

\section{Three-dimensional structure modeling}

187

For 10 out of the 797 nsLTP dataset, one or more experimentally determined 3D structures were available and downloaded from the Protein Data Bank (http://www.rcsb.org/pdb). Theoretical structures were calculated for the other 787 proteins using the @ tome2 suite of programs to perform homology modeling (Pons \& Labesse, 2009) (http://atome.cbs.cnrs.fr). The quality of each final structure model was evaluated using Qmean (Benkert et al., 2008). Structures with low quality (i.e. for which the cysteine scaffold could not be fully modeled) were discarded from further analysis (see Table 1).

\section{Structural classification}

All the remaining good-quality theoretical structures, together with the 10 experimental structures composed the 3D structure pool of the study. Except for the cysteine pattern analysis by ViTo, this structural pool was used in all further structural analysis. The structures were compared to each other in a sequence-independent manner, using the similarity matching method of the MAMMOTH program (Ortiz et al., 2002). The RMSD was calculated for each pair of structures, using the superposition between matched pairs that resulted in the lowest RMSD value. This superposition was computed using the Kabsch rotation matrix

202 (Kabsch, 1976; Kabsch, 1978) implemented in the MaxCluster program (Herbert A. 
203 unpublished). We used the RMSD score matrix calculated by MaxCluster as input for the

204 FastME program (Desper \& Gascuel, 2002) to calculate a structural distance tree.

205

206

3/ NsLTP functional annotation

207

Extensive bibliographic work was carried out to collect and classify functional information

208 available in the literature about the nsLTPs of the dataset. Gene Ontology (GO), Plant Ontology

209 (PO) and Trait Ontology (TO) terms were collected from the Gramene Ontologies Database

210 (http://www.gramene.org/plant_ontology) and organized in a dedicated database, together with the bibliographic references when available. The database was also enriched with additional information, such as methods used for gene expression studies (northern, RT-PCR or microarray

213 data, in situ hybridization), protein purification, in vitro or in planta antifungal and antibacterial activity, lipid binding or transport (fluorescence binding assay or in vitro lipid transfer). Information about tissues and organs used in cDNA libraries was collected from the NCBI databases (http://www.ncbi.nlm.nih.gov). A dataset with all the annotations is available online (http://dx.doi.org/10.18167/DVN1/1O5UAK.

218

\section{4/Integrative method 1: sequence -> structure -> function}

220

This method seeks to identify common ligand binding properties in nsLTPs clustered by

221 sequence similarity.

222

223

\section{Sequence consensus for each nsLTP type}

224

797 nsLTP sequences were clustered by type on the basis of regular expressions derived from the

225 consensus motifs described in (Boutrot et al., 2008). Each type subfamily was then aligned

226

227 individually and the resulting sequence profiles were globally aligned using MUSCLE. For each type subfamily, the most frequent amino acids were selected at each alignment position to build

228 the consensus sequence. A consensus amino acid was replaced by a gap if more than half of the

229 sequences were aligned with a deletion at the considered position.

230

NsLTP sequence-structure analysis using Frequently Aligned Symbol Tree (FAST)

232 An original tool was designed to highlight conserved amino acid positions specific to each

233 nsLTP phylogenetic type, and which might be decisive for their function. The algorithm relied

234 on a statistical analysis of each alignment row, after the sequences had been clustered according

235 to their phylogenetic distances.

236 For each type subfamily, the most frequent amino acids were selected at each alignment position

237 to build the consensus sequence. A consensus amino acid was replaced by a gap if more than half

238 of the sequences were aligned with a deletion at the considered position. We then calculated the

239 amino acid conservations and specificities over each column of the multiple sequence alignment

240 to delineate the functionally important residues in each nsLTP subfamily. This statistical analysis

241 is explained in Figure 3. 
242 In order to visualize the conserved and divergent regions of the sequences, different color ranges

243 were assigned to the nsLTP phylogenetic subfamilies. Conserved amino acid positions along the

244 whole alignment (CCP: Conserved Core Positions) are represented in grey/black, while

245 specifically conserved positions among proteins of the same subfamily (SDP: Specificity

246 Determining Positions) are represented in saturated colors corresponding to the family ones. The

247 tool enabled scrolling down of the alignment to easily identify both types of conserved positions

248 and two distant parts of the alignment could be displayed together to compare distant

249 phylogenetic subfamilies.

250 Contacts with ligands, solvent accessibility and other parameters could also be displayed above

251 the alignment. Using the Jmol interface, conserved amino acid residues could be projected on

252 nsLTP representative 3D structures, so that the potential role of each position could be

253 interpreted geometrically.

254

255

256

257

258

259

260

261

262

263

264

265

266

267

268

269

270

271

\section{5/Integrative method 2: function -> structure -> sequence}

Structural Trace Display is a method, based on Evolutionary Trace Display (ETD, Erdin et al.,2010), that seeks to identify common structural (1D, 3D) properties in nsLTPs sharing similar functions.

\section{Clustering of the structure tree}

As in a phylogenetic tree, nsLTPs in the structure tree were clustered according to their similarity. In the case of this particular tree, the similarity between nsLTPs was measured by a spatial distance in angströms (see paragraph 2/ NsLTP three-dimensional structures / Structural classification). Decreasing distance cutoffs ranged from $11.5 \AA$ (one cluster containing all nsLTP structures) to $0.5 \AA$. Each cutoff application caused a division of the tree into one or more subtrees that contained leaves (i.e. nsLTP structures) whose structural proximity altogether (represented by the pairwise RMSDs) was up to the value of the applied cutoff.

\section{InTreeGreat: an integrative tree visualization tool}

We developed an integrative tree visualization tool called InTreeGreat in order to display the whole or some parts of either sequence or structure distance trees.

272 InTreeGreat was implemented using PHP and Javascript, in order to generate and manipulate an

273 SVG graphical object.

274 The main objective of this tool is to graphically highlight correlations between 3D structures,

275 evolution, functional annotations or any available heterogeneous data. In the context of this

276 study, the interface was able to retrieve information from the nsLTP database to annotate the 277 tree.

278 InTreeGreat includes functionalities such as tree coloration, fading, and collapsing.

279 Heterogeneous data related to sequences (e.g. annotations, nsLTP classification) can be

280 displayed in colored boxes, aligned to the tree.

281

282 Cluster Selection 
283 Using InTreeGreat to investigate our annotated structure tree, we looked for clusters of nsLTPs

284 sharing the same kind of functional annotations. We focused our attention on one interesting

285 functional role highlighted in several nsLTPs: the implication in plant defense mechanisms

286 against pathogens (bacteria and/or fungus). In order to highlight structure-function relationships,

287 we studied three groups of nsLTPs (see Results section for details): (i) the "defense cluster" (43

288 proteins, distance cutoff $=1.5 \AA$ ); (ii) the cluster containing all type I fold proteins ( 402 proteins,

289 distance cutoff = $3 \AA$ ); (iii) a group manually composed of all type I fold nsLTPs for which a

290 functional role in defense and/or resistance against pathogens had been reported in the literature

291 (28 proteins).

292 Within each of these 3 clusters, the protein structure showing the shortest RMS distance from all

293 the others was selected as the representative structure of the cluster for the structural trace

294 calculation.

295

296

\section{Structural Trace calculation}

297 A structure-based sequence alignment was carried out on the nsLTP structures by Mustang

298 software (Konagurthu et al., 2006).

299 For each previously selected structural cluster, the corresponding set of protein sequences was

300 extracted from the multiple structural alignment of the nsLTPs. The Evolutionary Trace (ET)

301 method (Lichtarge et al., 1996) was applied: the partial multiple sequence alignment was

302 submitted as input for the ETC program (locally installed,

$303 \mathrm{http}: / /$ mammoth.bcm.tmc.edu/ETserver.html) together with the representative structure of the

304 cluster (selected as described in the previous paragraph).

305 The "evolutionary" traces based on the structural alignments corresponding to the three nsLTP

306 clusters were then compared to each other. To that end, the 30\% top-ranked residues of the

307 defense cluster trace were considered as constitutive of the reference trace (i.e. 27 most

308 conserved amino acid residues) and their ranking and scores in the two other traces were

309 analyzed. The results were compiled in a table and graphically visualized using PyMOL

310 (http://www.pymol.org/).

311

312

313 RESULTS

$314 \underline{1 / \text { NsLTP sequences analysis }}$

315

316 NsLTP dataset

317 Over the last four decades numerous proteins, whose ability to transfer lipids has not always

318 been demonstrated, have been annotated as nsLTPs on the basis of sequence similarity. In order

319 to understand more clearly the functional characteristics and the inner variability of this family,

320 we focused the study on the monodomain proteins, which present the strict and only nsLTP

321 domain, i.e. the eight-cysteine residues arranged in four disulfide bridges. In total, including the

322 wheat, rice and Arabidopsis sequences previously identified (Boutrot et al., 2008), together with

PeerJ reviewing PDF | (2019:04:36959:2:1:ACCEPTED 8 Jul 2019) 
323

324

325

326

327

328

329

330

331

332

333

334

335

336

337

338

339

340

341

342

343

344

345

346

347

348

349

350

351

352

353

354

355

356

357

358

359

360

361

362

sequences from the UniProt (Swiss-Prot/TrEMBL), NCBI and Phytozome databases, 797 nonredundant mature nsLTP sequences belonging to more than 120 plant species were kept for analysis. This first step allowed the selection of a relevant set of proteins covering variability in the nsLTP family. The number of sequences (797) was also large enough to challenge any analysis method we used during this study.

\section{Sequence alignment and Cysteine pattern}

The alignment of all non-redundant protein sequences for which the 3D structure was experimentally determined (10 sequences) was carried out twice, using the MUSCLE program on the one hand, and the MAFFT program on the other hand. The resulting alignments obtained with standard settings are shown on Figures $1 \mathrm{~A}$ and $1 \mathrm{~B}$.

In both cases, cysteine residues of the $8 \mathrm{CM}$ aligned quite well among the three represented types of nsLTPs (types I, II and IV), except for the Cys5-X-Cys6 (CXC) pattern region (where X stands for any amino acid residue). MUSCLE did align type I Cys5 with types II and IV Cys5', as well as type I Cys6 with types II and IV Cys6' (Figure 1A), just as previous studies typically showed (Liu et al., 2010; Siverstein et al., 2007). However, in the alignment carried out by MAFFT (Figure 1B), Cys5 of type I nsLTPs was equivalent to Cys6' of type II and IV nsLTPs, and not to the corresponding Cys5'.

To determine which of these two alignments better aligned nsLTP type I and II CXC patterns, we used ViTO program to compare the effects on the 3D structures of both sequence alignments. The 3D projection of MUSCLE sequence alignment (Figure 1C) showed that the attempt to spatially superimpose Cys5 and Cys6 of type I nsLTPs with Cys5' and Cys6' of type II nsLTPs respectively, was very expensive in term of Cys C-alpha RMSD value (7.32 $⿱$ Á).

On the contrary, according to the 3D projection of MAFFT sequence alignment (Figure 1D), type I Cys 5 could be well superimposed with type II Cys6', with a Cys C-alpha RMSD value dropping to $2.15 \AA$. Furthermore, type I hydrophylic X residue was exposed to the solvent, whereas type II apolar X residue was orientated toward the core of the protein, increasing the stability of the proteins. For these reasons we think that the sequence alignment calculated by MAFFT is more relevant.

This compound approach allowed us to sort the 797 sequences unambiguously into two main families.

\section{NsLTP sequence classification}

Our dataset was mainly composed of nsLTPs from angiosperm species (19 monocotyledonous species and 83 eudicotyledonous species) plus five gymnosperm species ( 35 sequences), one lycophyte species ( 34 sequences) and two bryophyte species (17 sequences). The monocot sequences were mainly represented by Poales nsLTPs ( 256 out of 270 sequences) whereas Rosid nsLTPs were the most abundant (364 out of 436 sequences) within eudicots.

The phylogenetic analysis showed that the pool of proteins clustered into nine different types, all highly supported (branch support $>0.84$ ). This result mostly confirmed Boutrot's classification, 
363 defined on A. thaliana, T. aestivum and $O$. sativa nsLTP sequences, in nine types (Boutrot et al., 364 2008). The main differences were the identification of a new group (named type XI), including 36523 sequences, and that Boutrot's type VII nsLTPs disappeared from our dataset. Indeed, the 366 latter did not satisfy the $8 \mathrm{CM}$ criteria as they have only seven cysteine residues in their 367 sequences. For the same reason, Wang's A, B, C and D types (Wang et al., 2012) were not 368 represented in our classification.

369

370

371

372

373

374

375

376

377

378

379

380

381

382

383

384

385

386

387

388

389

390

391

392

393

394

395

396

397

398

399

400

401
Type I nsLTPs formed a well-supported monophyletic group (branch support of 0.879) and predominated over the other types, as they accounted for more than half of our dataset (417 out of 797 sequences). This was also observed by Wang (Wang et al., 2012) with a set of 595 nsLTPs. Conversely, in Solanaceae, the most abundant nsLTPs belong to a type referred to as type $\mathrm{X}$ by Wang (70 out of 135 sequences) and which seems specific to that plant family (Liu et al., 2010) but was not present in our dataset. To avoid any confusion, we did not used type X denomination in this work. Type II nsLTPs were the second most abundant type (126 sequences) followed by type V (70 sequences) and type VI (60 sequences). Type IX (12 sequences) was mainly composed of Physcomitrella patens nsLTPs and type XI (23 sequences) was mainly composed of nsLTPs from eudicot species. Twelve nsLTPs were not included in any of the identified types: these were mainly $P$. patens (6 sequences) and $S$. moellendorfii (4 sequences) proteins (Figure 2).

Type XI were grouped in a cluster of 23 sequences in the phylogenetic tree, fairly well supported by a branch of 0.879 aLRT SH-like score. Type XI appeared between type I and the other types, but even though type XI and I were grouped together in the tree, it remained unclear which of the 3 groups (type I, type XI, and other types) diverged first.

All nsLTP types were represented in eudicots while types IX, X (in Wang's nomenclature) and XI were not identified in monocot species. Within the lycophyte and bryophyte species, no type II, III, IV nor VIII nsLTPs were identified. In the same way, no type III, VIII, IX or XI were identified within gymnosperm species. Ten out of the 16 moss $P$. patens nsLTPs were type IX, the other 6 remained un-typed, and the only liverwort Marchantia polymorpha nsLTP was a type VI. The 34 S. moellendorfii sequences were mainly types V and VI (15 and 7, respectively) and seven nsLTPs belonged to the new type XI. The $P$. patens and $S$. moellendorfii nsLTPs formed independent branches or were located at the same branch as type V in Wang's phylogenetic tree (Wang et al., 2012) and were included in type D in Edstam's classification (Edstam et al., 2011). However, Edstam's type D included rice and Arabidopsis type IV, V and VI nsLTPs. Edstam's type G nsLTPs, which corresponded to GPI-anchored LTPs and types J and K, which did not fit our molecular mass criteria or contain more than one $8 \mathrm{CM}$ motif were not included in our dataset.

Peer) reviewing PDF | (2019:04:36959:2:1:ACCEPTED 8 Jul 2019) 
402 According to Yi and coworkers (Yi et al., 2009), Allium nsLTPs may constitute a novel type of 403 nsLTPs harboring a C-terminal pro-peptide localized in endomembrane compartments. In the 404 prolamin superfamily tree of Radauer and Breiteneder (Radauer \& Breiteneder, 2007), the Allium 405 сера nsLTP (192_ALLCE) is closed but not included in the type I nsLTPs. In our phylogenetic 406 tree, the three nsLTPs from Allium species were classified as type I. The 501_MEDTR medicago 407 nsLTP was suggested to belong to a new nsLTP subfamily involved in lipid signaling (Pii et al., 408 2010) like Arabidopsis DIR1 (151_typeIV_ARATH). In our phylogenetic tree, both proteins 409 were identified as type IV nsLTPs.

410

The Theobroma cacao genome contains at least 46 nsLtp genes distributed across the ten chromosomes. Several T. cacao nsLtp genes are organized in clusters, as observed in the rice, Arabidopsis and sorghum genomes (Boutrot et al., 2008; Wang et al., 2012). Apart from nine sequences that were classified in the new type XI, all other T. cacao nsLTPs were classified within the previously identified types and belonged mainly to type I (14 sequences), type VI (7 sequences) and type V (6 sequences).

It is worth noting that all the nsLTPs identified as allergens (IgE binding) were type I, except one type II nsLTP (545_BRACM). The 501_MEDTR nsLTP was also suggested to play a role in the root nodulation process (Pii et al., 2009; Pii et al., 2013). Lipid signaling (lysophosphatidylcholine) has been reported to be involved in symbiosis (Bucher et al., 2009). This analysis was the most extensive so far and confirmed most of Boutrot's classification, but complements it due to a larger dataset and a more detailed phylogeny analysis.

\section{2/ NsLTP structure analysis}

426

427

\section{NsLTP structure modeling}

429

Given the nsLTP fold conservation and the quality of the available experimental structures, reliable models could be obtained for all nsLTPs using the comparative modeling method, although the sequence identity observed among all nsLTP sequences was only in the range of $25 \%$.

Models deduced by fold-recognition using the @TOME-2 server displayed overall good quality, as shown in Table 1 summarizing the Qmean scores. For 96\% of the models, Qmean scores were above 0.4 , and $57 \%$ of the models obtained scores ranging from 0.5 to 0.9 ., corresponding to scores for high-resolution proteins.

436 For 121 theoretical structures, the polypeptide chain could not be fully built and the resulting models were lacking at least one of the 8 cysteine residues. Such models were discarded and only the complementary pool of 677 structures was kept for further analysis.

439 All the structural alignments and three-dimensional models are available at: http://atome.cbs.cnrs.fr/AT2B/SERVER/LTP.html 


\section{NsLTP sequence - structure relationships}

443 In order to challenge the structure - function relationship analysis on such a big set, we decided 444 to develop a new tool called FAST, which builds consensus sequences for each family, and 445 highlights the sequence conservation and specificities on the alignment and the associated 3D 446 structures.

447 Figure 3 shows the consensus sequence alignment for all nsLTP types. The pool of 797 sequences was clustered by type on the basis of regular expressions derived from the consensus motifs described by Boutrot and coworkers (Boutrot et al., 2008). Each type subfamily was then aligned individually and the resulting sequence profiles were globally aligned using MUSCLE.

451

452

453

454

455

456

457

458

459

460

461

462

463

464

465

466

467

468

469

470

471

472

473

474

475

476

477

478

479

480

481
Many residues specifically conserved in type I nsLTP1 corresponded to important folding differences between type I nsLTPs on the one side and all other LTP types on the other side. In the following sections, we list type I nsLTP-specific residues whose differential conservation was supported by structural or experimental data.

First, Gly37, which was specifically conserved in type I nsLTPs, allowed very tight contact of helix 1 and helix 2, which were connected by the disulfide bridge Cys17-Cys34. The closest backbone distance between position 13 of helix 1 and position 37 of helix 2 was $3.34 \AA$ in a type I nsLTP structure (PDB code 1mid) while it was $6.45 \AA$ in a type II nsLTP structure (PDB code 1tuk). These increased helix distances closed the ligand tunnel, which was opened in type I nsLTPs between helix 1 and helix 3 , and created two distinct cavities separated by a septum in type II nsLTPs (Hoh et al., 2005). Larger distances between helix 1 and helix 2 were predicted in all nsLTP sequences where Gly37 was mutated into larger residues (i.e. all types but I and XI) and should cause major rearrangement of the ligand cavity entrance on this side of the proteins. Arginine and lysine residues at position 51 and bulky hydrophobic residues at positions 87 and 89 were two other conserved specificities among type I nsLTPs. The side chains at position 51 had type I-specific polar interactions with the ligand at the cavity entrance near the C-terminal loop, which were not found in other nsLTP types, as detailed later in Figure 4.

In addition, in type I nsLTPs, the 5th and 6th cysteine residues belonged to helix 3 and were bridged with the first and $8^{\text {th }}$ cysteines, respectively. These two-disulfide bridges tightened both sequence termini to the protein core. Conversely, in types II and IV nsLTPs, the 5th and 6th cysteines showed permuted bridging partners (to 8 th and $1^{\text {st }}$ cysteines, respectively). The intermediate residue connecting the 5th and 6th cysteines was exposed to solvent in type I nsLTPs, while it was replaced by a bulky hydrophobic residue interacting with the ligand in the type II and IV nsLTP core at position 54 of the alignment. It was shown by site-directed mutagenesis that the replacement of this intermediate residue by an alanine residue perturbed folding, ligand binding and lipid transfer activity in type II nsLTPs (Cheng et al., 2008). The position 54 in our alignment corresponds to residue 36 in Cheng's article. In the light of these experiments, it is therefore interesting to note that alanine residues were frequent at position 54

Peer) reviewing PDF | (2019:04:36959:2:1:ACCEPTED 8 Jul 2019) 
482 in type I nsLTPs, while larger hydrophobic residues almost always occupied this buried position 483 in other nsLTP types.

484

The mutation to alanine of the residue at position 63 (residue 45 in Cheng's article) was also shown experimentally to be destabilizing in type II nsLTPs (Cheng et al., 2008). This position was occupied by large hydrophobic residues in all nsLTPs but types I and V, where alanine residues were frequent, and type III, where it corresponded to a deletion of 12 consecutive residues. Other residues specifically conserved in type I nsLTPs were helix N-capping Thr6 and Thr47, whose side chains formed stabilizing hydrogen bonds with the protein backbone, and Tyr20, which was the center of a conserved hydrophobic cluster with Pro30 and Leu/Ile79. The interaction of Tyr20 with Pro30 was experimentally confirmed by the large up field shift of

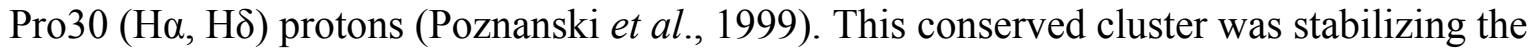
interface between helices 1 and 4, but did not participate in the ligand cavity. This particular helix interface was also observed in nsLTP types III, VI, VIII and XI.

We then analyzed the atomic interactions observed between type I nsLTPs and their associated ligands in 19 PDB structures (supplementary data). Most contacts involved hydrophobic side chains of the type I nsLTP proteins and carbons of the ligands. Marginally, the most frequent polar contacts involved the side chains of conserved arginines at position 46 of the type I nsLTP alignment, lysines at position 54, aspartic acids at position 90, and various polar atoms of histidines, lysines and asparagines at position 37. It should be stressed that none of these polar interactions were shared by more than $31 \%$ of the protein-ligand complexes (fewer than $6 / 19$ PDB structures) although the least similar protein pair from the 19 structure set shared $67 \%$ sequence identities. This low level of polar contact conservation in homologous proteins with very similar sequences clearly indicated that no specific polar interactions anchored the proteinligand complexes in particular conformations. From this statistical analysis of protein-ligand polar contacts that did not exhibit a preferential cavity region for the interaction with the ligand polar heads, it could not be concluded that there was a preferred ligand orientation in the type I

511 nsLTP tunnel. This observation was supported by recent protein-docking simulations and protein

512 binding evaluations, which also concluded on a lack of preferred orientations of the ligand in the

513 cavities of type I nsLTPs, and clear dominance of hydrophobic interactions in the protein-ligand

514 interface (Pacios et al., 2012).

515

516 Lastly, positions 82 to 94, which corresponded to the C-terminal loop, included some more 517 residues specifically conserved in nsLTPs. This loop was much longer in type I nsLTPs than in 518 other types, and had a major impact on the orientation of the ligand in the cavity, as shown in 519 Figure 4.

520

521 Conserved and specific residues in the nsLTP family 
522 The potential impact of variability within the nsLTP family on the tree dimensional structure of 523 the proteins was further investigated. As shown in Figure 4, the ligand cavity opening near the C524 Terminal loop was very different when we compared the nsLTP structures of type I versus those 525 of types II and IV. The C-terminal loops connected the 4 helices to the 3 helices through the 526 disulfide bridge between cysteine residues localized at alignment positions 95 and 55. Both 527 helices 2 and 3 and the C-terminal loop were longer in type I than in types II and IV nsLTPs. In 528 the type I nsLTPs, these elongations created a ligand cavity entrance along an axis perpendicular 529 to the figure plane, while in types II and IV nsLTPs, the entrance was approximately parallel 530 with the figure plane. Consequently, ligands would access the cavities on opposite sides of the C531 terminal loop in type I versus types II and IV nsLTPs. Helix 2 and 3 were extended by an extra 532 turn in type I nsLTPs comparatively to the structures of the other types. Moreover, the small 533 space left in between helices 2 and 3 and the C-term loop was capped in types II and IV by bulky 534 hydrophobic residues (Phe54 in 1tuk and Phe51 in 2rkn), while that position was occupied by a 535 positively charged lysine or arginine in type I nsLTPs (red colored Arg51 in 1mid), whose side 536 chain formed a hydrogen bond with the polar tail of the ligand.

537

The structural differences observed between type I nsLTPs versus types II and IV can be generalized to other nsLTP types by looking at the alignment of consensus sequences in Figure 3. First, the extension of helices 2 and 3 in type I nsLTPs corresponded to a 6- to 8-residue insertion in the consensus sequence alignment, which differentiated type I from every other type of nsLTPs. Secondly, the C-terminal loop connecting the last two cysteine residues was, on average, 13 residues long in type I nsLTPs, while this loop was shortened to 6, 6, 7, 12, 9, 8, 6 and 9 residues long in types II, III, IV, V, VI, VIII, IX and XI, respectively. Lastly, the capping hydrophic residues at positions 54 and 51 of types II and IV nsLTPs were also observed in all the other nsLTP types. These conserved differences between type I and other types of nsLTP sequences indicated with high confidence that the global fold of type I LTP differed from the fold of the other nsLTP types and that the ligand cavity entries in type I nsLTPs were uniquely located.

550 The fold of type I nsLTPs will be hereafter referred to as "Type-1 fold" and the alternative fold of Types II to XI will be referred to as "Type-2 fold". (in other words: Roman numerals I to XI correspond to phylogeny analysis while Arabic numerals 1 or 2 refer to structural analysis)

The preceding analysis of the evolutive conservations specific to type I nsLTPs revealed many residues whose role could be explained by local structural differences with the available types II and IV nsLTP structures. This comparative structure analysis confirmed the clear separation between type I and all the other nsLTP types initially observed in the phylogenetic tree inferred from a multiple sequence alignment of the 797 available proteins. The key residues were usually present in type I nsLTPs only and suggested that many structural differences observed when comparing type I versus types II and IV nsLTPs should also be observed versus other nsLTP types, particularly regarding ligand orientation and cavity entrances. This observation should 
562 guide the choice of templates when nsLTP types with unknown structures are modeled by

563 homology.

564

565

\section{Structure classification}

566

In order to correlate the evolution of protein sequences and the impact on the corresponding

567 structures, we produced a circular tree according to structural distances (Fig. 5). Whereas type I

568 remained together in this second classification, other phylogenetic types were relatively scattered in the tree. A majority of type II nsLTPs remained together in this tree, as was also the case for type IV and type III, but no clear and reliable segregation between all non-type I nsLTPs could be made. Looking at the 3D structures allowed us to confirm the hypothesis that only two major structural types could be distinguished. They will be hereafter referred to as "Type-1 fold" and "Type-2 fold". the residue standing between Cys5 and Cys6, being respectively hydrophilic in type I and apolar in type II proteins (Douliez et al., 2001; Marion et al., 2004). Based on the multiple sequence alignment of the 797 nsLTPs and observation of the nature of the central residue in the CXC pattern, together with the observations made in the preceding sequence-structure analysis, we suggest that types III, IV, V, VI, VIII, IX and XI nsLTP C5 and C6 residues will adopt the same spatial conformation as type II proteins, i.e. the "Type-2 fold".

581

582

\section{NsLTP structure-function relationship}

583

Dealing with big datasets can be cumbersome and requires a very efficient interface. To address this challenge, we developed InTreeGreat, a Javascript/PHP interface, compatible with every

585

586

587

588

589

590

591

592

593

594

595

596

597

598

599

600

601 standard web navigator. It is able to display and explore any tree and to deal with branch and leaf coloring, branch lengths, branch support (or any other branch labels), and can aggregate heterogeneous data (annotations, expression profiles, etc.). Figure 6 shows how InTreeGreat can be used to display phylogenic trees together with various types of annotations.

Among the annotated nsLTPs (433 out of 797), we focused on those that had been reported for their role in plant defense and/or resistance against pathogens (bacteria and/or fungi). To simplify, we shall hereafter refer to them as "defense nsLTPs" in the present discussion. By investigating structural similarities between the 31 identified defense nsLTPs in our annotated dataset, we attempted to identify key amino acid residues that would be good candidates for SDM experiments as they may bestow their functional properties on these proteins. Looking at the distribution of the defense nsLTPs in our structural classification (Figure 6) we observed that they were predominantly found in the type I part of the tree (28 proteins), with only 3 defense nsLTPs with a type II (85, 151, 501 - UniProtKB - P82900: Non-specific lipidtransfer protein 2G, Q8W453: Putative lipid-transfer protein DIR1, O24101: Lipid transfer protein). We therefore preferred to focus on the Type-1 fold nsLTPs and study the structural trace(s) inside this important subfamily of nsLTPs. 
602 The cluster containing all Type-1 fold defense nsLTPs corresponded to the whole type I part of

603 the tree (402 members). The corresponding structural trace was calculated, but it could not be

604 linked to the defense function, as the proportion of annotated nsLTPs with a defense function

605 was too low ( 28 out of 402 , i.e. $7 \%$ ).

606 In order to obtain a meaningful trace of the potential defense function, we needed to select a

607 cluster with a higher proportion of annotated defense nsLTPs. The best cluster we could find was

608 a relatively small cluster (43 members) of proteins with a structural distance no greater than $1.5 \AA$

609 (i.e. 1.5 cut off), which contained 33\% of the defense nsLTPs (i.e. 10 out of 31 proteins). This

610 cluster will be referred to as "defense cluster" in the further discussion.

611

612 The structural trace of the defense cluster showed several differences in comparison with the

613 structural trace of the Type-1 fold cluster (Table 2). Apart from the 8 Cys residues that were

614 common to all nsLTPs, the $30 \%$ top ranked (i.e. 27 residues) most conserved residues were not

615 the same, or did not come in the same order in both traces. According to the defense cluster

616 trace, residue Asp at position 259 of the alignment (Asp45 in protein 525) was as strongly

617 conserved as the 8 Cys residues. Residue Ile at position 402 (Ile80 in protein 525) was among the

6184 best ranked residues after the 8 Cys residues and obtained a very low coverage, variability and

619 rvET score. In terms of the ranking of these two (amino acid) residues in the Type-1 fold nsLTP

620 trace, they appeared to occur much later in the ranking (20th and 21st rank, respectively) with

621 much higher rvET scores and large variability in terms of the number and physico-chemical

622 properties of the residues (Table 2). It can be suggested that these two residues were not critical

623 for maintaining structure integrity, but could bestow functional specificity on the proteins

624 classified in the defense cluster. In the trace obtained for the group composed by all the other

625 Type-1 fold defense nsLTPs, both residues Asp and Ile were among the 4 best ranked residues

626 after the 8 Cys residues and also showed good coverage and rvEt scores (Table 2).

627

628

Three other residues located at positions 137, 154 and 266 of the structural alignment were

629 differently conserved in the three clusters. Interestingly, these three positions showed good

630 conservation ranking, but the variability of the three corresponding residues was notably higher

631 in the Type-1 fold cluster. Indeed, in the defense cluster trace, position 137 was occupied either

632 by a valine or by an alanine residue (Val7 in protein 525) and position 154 was occupied either

633 by a leucine or by a valine residue (Leu11 in protein 525). Thus, both positions were occupied by

634 hydrophobic residues in defense proteins, which was not always the case in Type-1 fold proteins

635 (Table 2). In the same way, position 266 was occupied either by an arginine or a lysine residue

636 (both positively charged residues) (Lys46 in protein 525) in defense proteins, but allowed greater

637 variability in terms of physicochemical properties in the other proteins harboring a Type-1 fold.

638 The fact that these three positions of the structural alignment belonged to the top $30 \%$ most

639 conserved among all Type-1 fold nsLTPs suggested their importance in these proteins. However,

640 because the variability at these three positions was very small among defense nsLTPs and

641 because the physico-chemical property was strongly conserved, we suspected that residues

642 located at positions 137, 154 and 266 of the structural alignment might bestow functional

643 specificity, at least in the case of defense/resistance proteins. 
644

645

646

647

648

649

650

651

652

653

654

655

656

657

658

659

660

661

662

663

664

665

666

667

668

669

670

671

672

673

674

675

676

\section{7}

678

679

680

681

682

683

Figure 7 shows the five residues highlighted in Table 2 in the 3D structural context of the representative protein of the defense cluster (protein 525). In this protein, conserved residues Asp and Ile were located at positions 45 and 80, respectively. The two small hydrophobic residues were Val7 and Leu11 and the positively charged residue was Lys46. All five key residues were located around the ligand cavity (Figure 7), which allowed either guidance or direct contact with the lipid. This observation was consistent with the suggested hypothesis.

NsLTP sequence-structure analyses using either FAST or STD revealed some key residues or key positions (in type I: Gly37, Arg/Lys51, bulky hydrophobic residues 87 and 89, Ala54, Thr6, Thr47, Tyr20, Pro30, Leu/Ile79, longer C-terminal loop; large hydrophobic residue 63 in types II, III, IV, VI, VIII, IX nsLTPs). The structural trace analysis highlighted other amino acid residues (in type I defense/resistance nsLTPs: Asp45, Ile80, Val/Ala7, Leu/Val11, Arg/Lys46). It is important to note that these two complementary analyses by FAST and STD were not meant to lead to the same kind of conclusions. Indeed, using sequence information projected on the 3D structure, the first method revealed nsLTP-type-specific amino acid residues that could be involved in structure stabilization and/or ligand interaction, given their structural context. The second method however considered a set of functionally close nsLTPs sharing a very similar structure and highlighted over-representatively conserved amino acid residues that might thus bestow functional specificity on these proteins. These two approaches took inverse directions in the path sequence - structure - function. The "sequence-to-function" method would lead to more precise conclusions if more data about the inner structural mechanisms of lipid binding were available (only a few structures of nsLTP-lipid complexes have been experimentally determined so far). The "function-to-sequence" method would give us a better overview of the range of nsLTP activities if the functional data were not so rare and heterogeneous.

\section{However, we assumed that this combination of approaches i) allowed structure-sequence} analysis for large multigene families, ii) could reveal structural patterns related to functions that were not revealed so far, as alignments would have been limited to primary sequences only and iii) allowed a comparison of groups composed of proteins with an evolutionary connection with groups displaying structural similarity.

\section{DISCUSSION}

A) We combined two powerful alignment algorithms (MAFFT and MUSCLE) together with a $3 \mathrm{D}$ projection of the impact of alignment on the structure of proteins (VITO). Real-time monitoring of the impact of gap positions and lengths on the resulting 3D model offered the possibility of discriminating between various alignment possibilities. This allowed us to provide definitive insight into the old debate about the CXC pattern and its implication for the structure

PeerJ reviewing PDF | (2019:04:36959:2:1:ACCEPTED 8 Jul 2019) 
684 of LTPs (Douliez et al. 2000). The resulting alignment allowed us to classify unambiguously all

685

686

687

688

689

690

691

692

693

694

695

696

697

698

699

700

701

702

703

704

705

706

707

708

709

710

711

712

713

714

715

716

717

718

719

720

721

722

723

724

797 sequences in main two nsLTP families.

B) The phylogenetic analysis was the most extensive to date, including 797 nsLTP sequences. This was a much more complete description than the previous one (195 sequences, Wang et al., 2012).

This phylogenetic analysis was conducted from a clearly defined dataset: sequences were selected using unambiguous parameters optimizing the quality of the output tree, also considering our 3D structural integration objective. Although GPI-Anchored LTP could have been included in this study, their incomplete homology with other LTPs and the lack of any experimental 3D structure, convinced us not to include them. Thanks to this choice, alignment quality was preserved, and a better-quality 3D structural model are used. This analysis allowed us to classify unambiguously all 797 sequences in the main two nsLTP families, complementing and reinforcing the former classification by Boutrot (Boutrot et al. 2008).

C) The production of more than 600 3D structural models and the collection of numerous functional annotations enabled progress to be made in the study of structure-function relationships of nsLTPs. The re-use of the ETD method in a close and adapted form (STD) led to the identification of amino acids involved in the functional specialization of some nsLTPs. STD allowed us to highlight amino acids specific to certain functions. One of the limiting points of this analysis remained the publication bias. Indeed, the annotations were not evenly distributed among available sequences, nor was it possible to distinguish between an unsearched function and a function not found. It seemed difficult to propose a solution to circumvent this bias (Douliez et al. 2000).

D) The structure tree clearly showed that all Type I ns-LTPs adopted the same folding (Type-1 fold), while all the other proteins adopted the second fold (Type-2 fold). This approach seemed very interesting but did not offer the same level of detail and the same analytical power as the phylogenetic approach. This was understandable, because phylogeny compares the different proteins with a much larger number of parameters (site-to-site mutation, classification of sites by mutation rate, use of refined distance matrix, etc.) while the structure tree only uses the RMSD of the structures taken 2 by 2 . While this innovative information was very interesting, it could potentially be improved if we had templates from each sub-family for the generation of molecular models (experimental structures are available for Type I, II and IV). Indeed, at this level of analysis, it is conceivable that models obtained from experimental structures for the other types (III, V, VI, VII, VIII, IX and XI) would provide improved models allowing the detection of other key residues.

\section{CONCLUSIONS}


725 Plant non-specific Lipid Transfer Proteins constitute a complex family of proteins whose

726 biological functions are far from well understood. However, it has become clear for years that

727 they are of increasing interest for agronomical and nutritional issues.

728 Experimental approaches are irreplaceable for accessing their inner functional mechanisms.

729 However, such methods are expensive and time-consuming. Furthermore, they produce a large

730 amount of heterogeneous data. For all these reasons, resorting to bioinformatics methods has

731 long become necessary to organize and analyze existing data, and/or model and hypothesize new

732 data.

733 This paper presented a new methodology based on the combination of either classical or original

734 bioinformatics approaches, using various computational tools to extract information and suggest

735 new hypotheses from a large pool of experimental data about the plant nsLTP superfamily of

736 proteins.

737 In this paper, we:

738 1) Suggested a new definition of the nsLTP superfamily, with a set of criteria based on sequence

739 length, sequence composition (e.g. Cys involved in SS bonds) and structure (monodomain).

740 2) Confirmed and enriched Boutrot's phylogenetic classification of plant nsLTP sequences.

741 3) Demonstrated the need for a small shift in the CXC alignment that reflected the existence of

742 two main distinct nsLTP folds.

743 4) Calculated 666 good quality theoretical three-dimensional structures of nsLTPs.

744 5) Developed an original alignment tool to detect conserved and specific positions among the

745 different phylogenetic types of nsLTPs.

746 6) Used the latter tool to reveal some key residues.

747 7) Suggested a new structure-based classification of the 676 nsLTP structures now available (10

748 experimental +666 theoretical), which that allow clustering by structural similarity.

749 8) Annotated all available information about the function.

750 9) Developed an original interface allowing quick visualization of several types of annotations 751 on any phylogenetic tree.

752 10) Revealed, using structural trace analysis, potential specific amino acid residues involved in

753 plant defense and/or resistance against pathogens

754

755 Our work was made more difficult by the problems of annotation bias for which we did not

756 expect a practical solution. However, it seemed that some of our results could be improved if we

757 had additional experimental structures for all types of nsLTPs.

758 To researchers who may not grasp the importance of the protein structure-function relationships

759 we would like to insist on three main contributions of the methods presented in this work:

760 - The structural classification agrees with the sequence classification by phylogenetic types.

761 - The sequence-structure analysis highlights key-residues explaining the specificities of the

762 different folds.

763 - The structure-function analysis based on the evolutionary trace of the aligned sequences can

764 show the functional signature of a subfamily of proteins. 
765 Furthermore, the structural dichotomy between type I nsLTPs and all the others may go

766

767

768

769

770

771

772

773

774

775

776

777

778

779

780

781

782

783

784

785

786

787

788

789

790

791

792

793

794

795

796

797

798

799

800

801

802

803

804

unnoticed by anyone who would focus on one sequence and who would not conduct a combined analysis on a large set of sequences. We encourage researchers studying nsLTPs to use this approach combining sequence alignment, phylogeny and structural biochemistry. This would enhance the power of the analysis by drawing a connection between primary sequence, threedimensional structure and function.

More broadly, we consider that this type of combined approach should be favored for any study involving a multigenic family.

\section{ACKNOWLEDGEMENTS}

This work was supported by the CIRAD - UMR AGAP HPC Data Centre of the South Green Bioinformatics platform.

The authors are thankful to Dr. Franck Molina for his key role at the beginning of this project and all the fruitful and friendly discussions.

We are thankful to Peter Biggins for the careful and critical review of this manuscript.

\section{REFERENCES}

Argout, X., Salse, J., Aury, J-M., Guiltinan, M.J.,Droc, G., Gouzy, J., Allegre, M., Chaparro, C., Legavre, T., Maximova, S.N., Abrouk, M., Murat, F., Fouet, O., Poulain, J., Ruiz, M., Roguet, Y., Rodier-Goud, M., Barbosa-Neto, J.F., Sabot, F., Kudrna, D., Ammiraju, J.S.S., Schuster, S.C., Carlson, J.E., Sallet, E., Schiex, T., Dievart, A., Kramer, M., Gelley, L., Shi, Z. Bérard, A., Viot, C., Boccara, M., Risterucci, A-M. Guignon, V., Sabau, X., Axtell, M.J. Ma, Z., Zhang, Y., Brown, S., Bourge, M., Golser, W., Song, X., Clement, D., Rivallan, R., Tahi, M., Akaza, J.M., Pitollat, B., Gramacho, K., D’Hont, A., Brunel, D., Infante, D., Kebe, I., Costet, P., Wing, R., McCombie, W.R., Guiderdoni, E., Quetier, F., Panaud, O., Wincker, P., Bocs, S., and Lanaud, C. (2011). The genome of Theobroma cacao. Nat. Genet. 43, 101-108.

Arondel, V., Vergnolle, C., Cantrel, C., and Kader, J. (2000). Lipid transfer proteins are encoded by a small multigene family in Arabidopsis thaliana. Plant Sci. Int. J. Exp. Plant Biol. 157, 1-12. Aziz, N., Paiva, N.L., May, G.D., and Dixon, R.A. (2005). Transcriptome analysis of alfalfa glandular trichomes. Planta 221, 28-38.

Bendtsen, J.D., Nielsen, H., von Heijne, G., and Brunak, S. (2004). Improved prediction of signal peptides: SignalP 3.0. J. Mol. Biol. 340, 783-795.

Benkert, P., Tosatto, S.C.E., and Schomburg, D. (2008). QMEAN: A comprehensive scoring function for model quality assessment. Proteins 71, 261-277.

Benkert, P., Biasini, M., and Schwede, T. (2011). Toward the estimation of the absolute quality of individual protein structure models. Bioinforma. Oxf. Engl. 27, 343-350. 
805 Berman, H.M., Westbrook, J., Feng, Z., Gilliland, G., Bhat, T.N., Weissig, H., Shindyalov, I.N., 806 and Bourne, P.E. (2000). The Protein Data Bank. Nucleic Acids Res. 28, 235-242.

807 Boutrot, F., Guirao, A., Alary, R., Joudrier, P., and Gautier, M.-F. (2005). Wheat non-specific

808 lipid transfer protein genes display a complex pattern of expression in developing seeds.

809 Biochim. Biophys. Acta 1730, 114-125.

810 Boutrot, F., Chantret, N., and Gautier, M.-F. (2008). Genome-wide analysis of the rice and

811 Arabidopsis non-specific lipid transfer protein (nsLtp) gene families and identification of wheat

812 nsLtp genes by EST data mining. BMC Genomics 9, 86.

813 Bucher, M., Wegmüller, S., and Drissner, D. (2009). Chasing the structures of small molecules

814 in arbuscular mycorrhizal signaling. Curr. Opin. Plant Biol. 12, 500-507.

815 Buhot, N., Douliez, J.P., Jacquemard, A., Marion, D., Tran, V., Maume, B.F., Milat, M.L.,

816 Ponchet, M., Mikès, V., Kader, J.C., and Blein J-P. (2001). A lipid transfer protein binds to a

817 receptor involved in the control of plant defence responses. FEBS Lett. 509, 27-30.

818 Buhot, N., Gomès, E., Milat, M.-L., Ponchet, M., Marion, D., Lequeu, J., Delrot, S., Coutos-

819 Thévenot, P., and Blein, J.-P. (2004). Modulation of the biological activity of a tobacco LTP1 by 820 lipid complexation. Mol. Biol. Cell 15, 5047-5052.

821 Cameron, K.D., Teece, M.A., and Smart, L.B. (2006). Increased accumulation of cuticular wax

822 and expression of lipid transfer protein in response to periodic drying events in leaves of tree

823 tobacco. Plant Physiol. 140, 176-183.

824 Cammue, B.P., Thevissen, K., Hendriks, M., Eggermont, K., Goderis, I.J., Proost, P., Van

825 Damme, J., Osborn, R.W., Guerbette, F., and Kader, J.C. (1995). A potent antimicrobial protein

826 from onion seeds showing sequence homology to plant lipid transfer proteins. Plant Physiol. 109,

$827 \quad 445-455$.

828 Catherinot, V., and Labesse, G. (2004). ViTO: tool for refinement of protein sequence-structure

829 alignments. Bioinforma. Oxf. Engl. 20, 3694-3696.

830 Chae, K., and Lord, E.M. (2011). Pollen tube growth and guidance: roles of small, secreted

831 proteins. Ann. Bot. 108, 627-636.

832 Chae, K., Kieslich, C.A., Morikis, D., Kim, S.-C., and Lord, E.M. (2009). A gain-of-function

833 mutation of Arabidopsis lipid transfer protein 5 disturbs pollen tube tip growth and fertilization.

834 Plant Cell 21, 3902-3914.

835 B. Chanda, Ye Xia, M. Kumar Mandal, K. Yu, K-T. Sekine, Q. Gao, D. Selote, Y. Hu, A.

836 Stromberg, D. Navarre, A. Kachroo and P. Kachroo. (2011). Glycerol-3-phosphate is a critical

837 mobile inducer of systemic immunity in plants. Nat. Genet. 43, 421-427.

838 Cheng, C.-S., Chen, M.-N., Lai, Y.-T., Chen, T., Lin, K.-F., Liu, Y.-J., and Lyu, P.-C. (2008).

839 Mutagenesis study of rice nonspecific lipid transfer protein 2 reveals residues that contribute to

840 structure and ligand binding. Proteins 70, 695-706.

841 Choi, Y.E., Lim, S., Kim, H.-J., Han, J.Y., Lee, M.-H., Yang, Y., Kim, J.-A., and Kim, Y.-S.

842 (2012). Tobacco NtLTP1, a glandular-specific lipid transfer protein, is required for lipid

843 secretion from glandular trichomes. Plant J. Cell Mol. Biol. 70, 480-491. 
844 Clark, A.M., and Bohnert, H.J. (1999). Cell-specific expression of genes of the lipid transfer

845

846

847

848

849

850

851

852

853

854

855

856

857

858

859

860

861

862

863

864

865

866

867

868

869

870

871

872

873

874

875

876

877

878

879

880

881

882

883

protein family from Arabidopsis thaliana. Plant Cell Physiol. 40, 69-76.

Cong, Q., and Grishin, N.V. (2012). MESSA: MEta-Server for protein Sequence Analysis. BMC Biol. 10, 82 .

D’Agostino, N. ,Buonanno, M., Ayoub, J., Barone, A., Monti, S. M., Rigano, Maria M. (2019). Identification of non-specific Lipid Transfer Protein gene family members in Solanum lycopersicum and insights into the features of Sola 13 protein. Sci. Reports. 9. 1607.Debono, A., Yeats, T.H., Rose, J.K.C., Bird, D., Jetter, R., Kunst, L., and Samuels, L. (2009). Arabidopsis LTPG is a glycosylphosphatidylinositol-anchored lipid transfer protein required for export of lipids to the plant surface. Plant Cell 21, 1230-1238.

Desper, R., and Gascuel, O. (2002). Fast and accurate phylogeny reconstruction algorithms based on the minimum-evolution principle. J. Comput. Biol. J. Comput. Mol. Cell Biol. 9, 687-705. Douliez, J.-P., Michon, T., Elmorjani, K., \& Marion, D. (2000). Mini Review: Structure, Biological and Technological Functions of Lipid Transfer Proteins and Indolines, the Major Lipid Binding Proteins from Cereal Kernels. Journal of Cereal Science, 32(1), 1-20.

\section{Douliez, J.P., Pato, C., Rabesona, H., Mollé, D., and Marion, D. (2001b). Disulfide bond} assignment, lipid transfer activity and secondary structure of a 7-kDa plant lipid transfer protein, LTP2. Eur. J. Biochem. 268, 1400-1403.

Douliez, J.P., Jégou, S., Pato, C., Mollé, D., Tran, V., and Marion, D. (2001a). Binding of two mono-acylated lipid monomers by the barley lipid transfer protein, LTP1, as viewed by fluorescence, isothermal titration calorimetry and molecular modelling. Eur. J. Biochem. 268, 384-388.

Dufayard, J.-F., Duret, L., Penel, S., Gouy, M., Rechenmann, F., and Perrière, G. (2005). Tree pattern matching in phylogenetic trees: automatic search for orthologs or paralogs in homologous gene sequence databases. Bioinforma. Oxf. Engl. 21, 2596-2603.

Edgar, R.C. (2004). MUSCLE: a multiple sequence alignment method with reduced time and space complexity. BMC Bioinformatics 5, 113.

Edstam, M.M., Viitanen, L., Salminen, T.A., and Edqvist, J. (2011). Evolutionary history of the non-specific lipid transfer proteins. Mol. Plant 4, 947-964.

Erdin, S., Ward, R.M., Venner, E., and Lichtarge, O. (2010). Evolutionary trace annotation of protein function in the structural proteome. J. Mol. Biol. 396, 1451-1473.

Gao, M., Yang, F., Zhang, L., Su, Z., and Huang, Y. (2017). Exploring the sequence-structurefunction relationship for the intrinsically disordered $\beta \gamma$-crystallin Hahellin. J. Biomol. Struct. Dyn. 1-11.

Ge, X., Chen, J., Sun, C., Cao, K. (2003). Preliminary study on the structural basis of the antifungal activity of a rice lipid transfer protein. Protein Eng. 6, 387-390.

Girault, T., François, J., Rogniaux, H., Pascal, S., Delrot, S., Coutos-Thévenot, P., and Gomès, E. (2008). Exogenous application of a lipid transfer protein-jasmonic acid complex induces protection of grapevine towards infection by Botrytis cinerea. Plant Physiol. Biochem. PPB 46, 140-149.

PeerJ reviewing PDF | (2019:04:36959:2:1:ACCEPTED 8 Jul 2019) 
884 Guindon, S., Dufayard, J.-F., Lefort, V., Anisimova, M., Hordijk, W., and Gascuel, O. (2010).

885 New algorithms and methods to estimate maximum-likelihood phylogenies: assessing the

886 performance of PhyML 3.0. Syst. Biol. 59, 307-321.

887 Henikoff, S., and Henikoff, J.G. (1994). Position-based sequence weights. J. Mol. Biol. 243,

$888 \quad 574-578$.

889 Herbert A, unpublished. Max Cluster. Available

890 at http://www.sbg.bio.ic.ac.uk/maxcluster (accessed 2019-08/08).

891 Hoh, F., Pons, J.-L., Gautier, M.-F., de Lamotte, F., and Dumas, C. (2005). Structure of a

892 liganded Type-2 non-specific lipid-transfer protein from wheat and the molecular basis of lipid

893 binding. Acta Crystallogr. D Biol. Crystallogr. 61, 397-406.

894 Huson, D.H., and Scornavacca, C. (2012). Dendroscope 3: an interactive tool for rooted

895 phylogenetic trees and networks. Syst. Biol. 61, 1061-1067.

896 Jang, C.S., Jung, J.H., Yim, W.C., Lee, B.-M., Seo, Y.W., and Kim, W. (2007). Divergence of

897 genes encoding non-specific lipid transfer proteins in the poaceae family. Mol. Cells 24, 215-

898223.

899 José-Estanyol, M., Gomis-Rüth, F.X., and Puigdomènech, P. (2004). The eight-cysteine motif, a

900 versatile structure in plant proteins. Plant Physiol. Biochem. PPB 42, 355-365.

901 Kader, J.-C. (1996). LIPID-TRANSFER PROTEINS IN PLANTS. Annu. Rev. Plant Physiol.

902 Plant Mol. Biol. 47, 627-654.

903 Kader, J.C., Julienne, M., and Vergnolle, C. (1984). Purification and characterization of a

904 spinach-leaf protein capable of transferring phospholipids from liposomes to mitochondria or

905 chloroplasts. Eur. J. Biochem. 139, 411-416.

906 Katoh, K., and Toh, H. (2010). Parallelization of the MAFFT multiple sequence alignment

907 program. Bioinforma. Oxf. Engl. 26, 1899-1900.

908 Katoh, K., Misawa, K., Kuma, K., and Miyata, T. (2002). MAFFT: a novel method for rapid

909 multiple sequence alignment based on fast Fourier transform. Nucleic Acids Res. 30, 3059-3066.

910 Konagurthu, A.S., Whisstock, J.C., Stuckey, P.J., and Lesk, A.M. (2006).

911 : a multiple structural alignment algorithm. Proteins 64, 559-574.

912 Kurowski, M.A., and Bujnicki, J.M. (2003). GeneSilico protein structure prediction meta-server.

913 Nucleic Acids Res. 31, 3305-3307.

914 Lange, B.M., Wildung, M.R., Stauber, E.J., Sanchez, C., Pouchnik, D., and Croteau, R. (2000).

915 Probing essential oil biosynthesis and secretion by functional evaluation of expressed sequence

916 tags from mint glandular trichomes. Proc. Natl. Acad. Sci. U. S. A. 97, 2934-2939.

917 Li, F., Fan, K., Ma, F., Yue, E., Bibi, N., Wang, M., Shen, H., Hasan, Md., Wang, X. (2016).

918 Genomic Identification and Comparative Expansion Analysis of the Non-Specific Lipid Transfer

919 Protein Gene Family in Gossypium. Sci Reports. 6. 1-15.

920 Li, X., Gasic, K., Cammue, B., Broekaert, W., and Korban, S.S. (2003). Transgenic rose lines

921 harboring an antimicrobial protein gene, Ace-AMP1, demonstrate enhanced resistance to

922 powdery mildew ( Sphaerotheca pannosa). Planta 218, 226-232.

923 Lichtarge, O., Bourne, H.R., and Cohen, F.E. (1996). An evolutionary trace method defines

924 binding surfaces common to protein families. J. Mol. Biol. 257, 342-358. 
925 Liu, W., Huang, D., Liu, K., Hu, S., Yu, J., Gao, G., and Song, S. (2010). Discovery,

926 identification and comparative analysis of non-specific lipid transfer protein (nsLtp) family in

927 Solanaceae. Genomics Proteomics Bioinformatics 8, 229-237.

928 van Loon, L.C., Rep, M., and Pieterse, C.M.J. (2006). Significance of inducible defense-related

929 proteins in infected plants. Annu. Rev. Phytopathol. 44, 135-162.

930 Maghuly, F., Borroto-Fernandez, E.G., Khan, M.A., Herndl, A., Marzban, G., and Laimer, M.

931 (2009). Expression of calmodulin and lipid transfer protein genes in Prunus incisa x serrula

932 under different stress conditions. Tree Physiol. 29, 437-444.

933 Maldonado, A.M., Doerner, P., Dixon, R.A., Lamb, C.J., and Cameron, R.K. (2002). A putative

934 lipid transfer protein involved in systemic resistance signalling in Arabidopsis. Nature 419, 399-

935403.

936 Meng, C. Yan, Y., Liu, Z., Chen, L., Zhang, Y., Li, X., Wu, L., Zhang, G., Wang, X., Ma, Z.

937 (2018). Systematic Analysis of Cotton Non-specific Lipid Transfer Protein Family Revealed a

938 Special Group That Is Involved in Fiber Elongation. Front. in Plant Sci. 9. 1285.

939 Molina, A., and García-Olmedo, F. (1993). Developmental and pathogen-induced expression of

940 three barley genes encoding lipid transfer proteins. Plant J. Cell Mol. Biol. 4, 983-991.

941 Mollet, J.C., Park, S.Y., Nothnagel, E.A., and Lord, E.M. (2000). A lily stylar pectin is necessary

942 for pollen tube adhesion to an in vitro stylar matrix. Plant Cell 12, 1737-1750.

943 Murzin, A.G., Brenner, S.E., Hubbard, T., and Chothia, C. (1995). SCOP: a structural

944 classification of proteins database for the investigation of sequences and structures. J. Mol. Biol.

$945247,536-540$.

946 Nieuwland, J., Feron, R., Huisman, B.A.H., Fasolino, A., Hilbers, C.W., Derksen, J., and

947 Mariani, C. (2005). Lipid transfer proteins enhance cell wall extension in tobacco. Plant Cell 17,

948 2009-2019.

949 Orengo, C.A., Michie, A.D., Jones, S., Jones, D.T., Swindells, M.B., and Thornton, J.M. (1997).

950 CATH--a hierarchic classification of protein domain structures. Struct. Lond. Engl. 1993 5,

951 1093-1108.

952 Ortiz, A.R., Strauss, C.E.M., and Olmea, O. (2002). MAMMOTH (matching molecular models

953 obtained from theory): an automated method for model comparison. Protein Sci. Publ. Protein

954 Soc. 11, 2606-2621.

955 Ovchinnikov, S., Park, H., Varghese, N., Huang, P.-S., Pavlopoulos, G.A., Kim, D.E.,

956 Kamisetty, H., Kyrpides, N.C., and Baker, D. (2017). Protein structure determination using

957 metagenome sequence data. Science 355, 294-298.

958 Liu F, Zhang X, Lu C, Zeng X, Li Y, Fu D, Wu G. Non-specific lipid transfer proteins in plants:

959 presenting new advances and an integrated functional analysis. J Exp. Bot. (2015)

960 Pacios, L.F., Gómez-Casado, C., Tordesillas, L., Palacín, A., Sánchez-Monge, R., and Díaz-

961 Perales, A. (2012). Computational study of ligand binding in lipid transfer proteins: Structures,

962 interfaces, and free energies of protein-lipid complexes. J. Comput. Chem. 33, 1831-1844.

963 Park, S.Y., Jauh, G.Y., Mollet, J.C., Eckard, K.J., Nothnagel, E.A., Walling, L.L., and Lord,

964 E.M. (2000). A lipid transfer-like protein is necessary for lily pollen tube adhesion to an in vitro

965 stylar matrix. Plant Cell 12,151-164. 
966 Pii, Y., Astegno, A., Peroni, E., Zaccardelli, M., Pandolfini, T., and Crimi, M. (2009). The 967 Medicago truncatula N5 gene encoding a root-specific lipid transfer protein is required for the 968 symbiotic interaction with Sinorhizobium meliloti. Mol. Plant-Microbe Interact. MPMI 22, $969 \quad 1577-1587$.

970 Pii, Y., Molesini, B., and Pandolfini, T. (2013). The involvement of Medicago truncatula non971 specific lipid transfer protein N5 in the control of rhizobial infection. Plant Signal. Behav. 8, 972 e24836.

973 Pons, J.-L., and Labesse, G. (2009). @TOME-2: a new pipeline for comparative modeling of 974 protein-ligand complexes. Nucleic Acids Res. 37, W485-491.

975 Poznanski, J., Sodano, P., Suh, S.W., Lee, J.Y., Ptak, M., and Vovelle, F. (1999). Solution 976 structure of a lipid transfer protein extracted from rice seeds. Comparison with homologous 977 proteins. Eur. J. Biochem. 259, 692-708.

978 Pyee, J., and Kolattukudy, P.E. (1995). The gene for the major cuticular wax-associated protein 979 and three homologous genes from broccoli (Brassica oleracea) and their expression patterns.

980 Plant J. Cell Mol. Biol. 7, 49-59.

981 Pyee, J., Yu, H., and Kolattukudy, P.E. (1994). Identification of a lipid transfer protein as the 982 major protein in the surface wax of broccoli (Brassica oleracea) leaves. Arch. Biochem. Biophys. 983 311, 460-468.

984 Qin, X., Liu, Y., Mao, S., Li, T., Wu, H., Chu, C., and Wang, Y. (2011). Genetic transformation 985 of lipid transfer protein encoding gene in Phalaenopsis amabilis to enhance cold resistance.

986 Euphytica 177, 33-43.

987 Radauer, C., and Breiteneder, H. (2007). Evolutionary biology of plant food allergens. J. Allergy 988 Clin. Immunol. 120, 518-525.

989 Radauer, C., Bublin, M., Wagner, S., Mari, A., and Breiteneder, H. (2008). Allergens are

990 distributed into few protein families and possess a restricted number of biochemical functions. J.

991 Allergy Clin. Immunol. 121, 847-852.e7.

992 G. J. Rocklin, T. M. Chidyausiku, I. Goreshnik, A. Ford, S. Houliston, A. Lemak, L. Carter, R.

993 Ravichandran, V. K. Mulligan, A. Chevalier, C. H. Arrowsmith, and D. Baker (2017). Global

994 analysis of protein folding using massively parallel design, synthesis, and testing. Science 357,

$995168-175$.

996 Salcedo, G., Sánchez-Monge, R., Barber, D., and Díaz-Perales, A. (2007). Plant non-specific

997 lipid transfer proteins: an interface between plant defence and human allergy. Biochim. Biophys.

998 Acta 1771, 781-791.

999 Sawano, Y., Hatano, K., Miyakawa, T., Komagata, H., Miyauchi, Y., Yamazaki, H., and

1000 Tanokura, M. (2008). Proteinase inhibitor from ginkgo seeds is a member of the plant

1001 nonspecific lipid transfer protein gene family. Plant Physiol. 146, 1909-1919.

1002 Silverstein, K.A.T., Moskal, W.A., Wu, H.C., Underwood, B.A., Graham, M.A., Town, C.D., 1003 and VandenBosch, K.A. (2007). Small cysteine-rich peptides resembling antimicrobial peptides 1004 have been under-predicted in plants. Plant J. Cell Mol. Biol. 51, 262-280. 
1005 Sror, H.A.M., Tischendorf, G., Sieg, F., Schmitt, J.M., and Hincha, D.K. (2003). Cryoprotectin 1006 protects thylakoids during a freeze-thaw cycle by a mechanism involving stable membrane 1007 binding. Cryobiology 47, 191-203.

1008 Sterk, P., Booij, H., Schellekens, G.A., Van Kammen, A., and De Vries, S.C. (1991). Cell1009 specific expression of the carrot EP2 lipid transfer protein gene. Plant Cell 3, 907-921.

1010 Sun, J.-Y., Gaudet, D.A., Lu, Z.-X., Frick, M., Puchalski, B., and Laroche, A. (2008).

1011 Characterization and antifungal properties of wheat nonspecific lipid transfer proteins. Mol.

1012 Plant-Microbe Interact. MPMI 21, 346-360.

1013 Thoma, S., Kaneko, Y., and Somerville, C. (1993). A non-specific lipid transfer protein from

1014 Arabidopsis is a cell wall protein. Plant J. Cell Mol. Biol. 3, 427-436.

1015 Trevino, M.B., and OConnell, M.A. (1998). Three drought-responsive members of the

1016 nonspecific lipid-transfer protein gene family in Lycopersicon pennellii show different

1017 developmental patterns of expression. Plant Physiol. 116, 1461-1468.

1018 Wang, H.W., Hwang, S.-G., Karuppanapandian, T., Liu, A., Kim, W., and Jang, C.S. (2012a).

1019 Insight into the molecular evolution of non-specific lipid transfer proteins via comparative

1020 analysis between rice and sorghum. DNA Res. Int. J. Rapid Publ. Rep. Genes Genomes 19, 1791021194.

1022 Wang, N.-J., Lee, C.-C., Cheng, C.-S., Lo, W.-C., Yang, Y.-F., Chen, M.-N., and Lyu, P.-C.

1023 (2012b). Construction and analysis of a plant non-specific lipid transfer protein database 1024 (nsLTPDB). BMC Genomics 13 Suppl 1, S9.

1025 


\section{Figure 1}

Effect of alternate cysteine residue alignments on the superposition of type I and II nsLTP experimentally determined structures.

(A) Common alignment of Cys5 (type I), Cys5' (types II and IV) (green) and Cys6 (type I), Cys6' (types II and IV) (magenta) of nsLTP sequences generated by MUSCLE. Only nsLTPs (PDB IDs) with known experimental structures were considered. (C) 3D projection of this alignment leads to an RMSD of $7.32 \AA$ between type I (blue backbone) Cys 6 and type II (pink backbone) Cys6', colorized as in (A). (B) Type I, II and IV nsLTP alignment generated by the MAFFT program, suggesting that type I Cys5 (dark green) corresponds to type II Cys6' (light green) rather than type II Cys5'. (D) 3D projection of this alignment leads to an RMSD of 2.15 $\AA$ between type I Cys5 and type II Cys6', colorized as in (B). Note that type IV nsLTPs are structurally close to type II nsLTPS. 


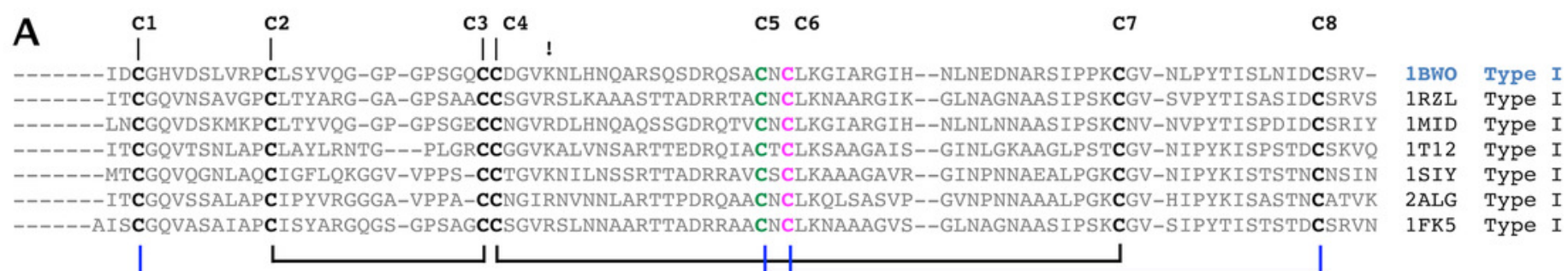

--------ACOASOLAV--CASAILS-GA-KPSGECCGNLR-----------AOOGCFCOYAKDPTYG-OYIRSPHARDTLTSCGL-AVPH-------C---- 1TUK TYPe II GVVGVAGAGCNAGQLTV--CTGAIAG-GA-RPTAACCSSLR----------AQQGCFCQFAKDPRYG-RYVNSPNARKAVSSCGI-ALPT-------CH--- 1L6H TYPe II ------IDLCGMSQDELNECKPAVSKENPTSPSQPCCTALQH---------ADFACLCGYKNSPWLGSFGVDPLASALPKOCGLANAPT--------C---- 2RKN TYPe IV

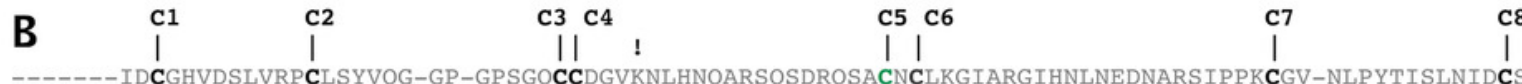
Lــ STKGV-SVPYTISASIDCSRVS ------LNCGQVDSKMKPCLTYVQG-GP-GPSGECCNGVRDLHNQAQSSGDRQTVCNCLKGIARGIHNLNLNNAASIPSKCNV-NVPYTISPDIDCSRIY -------ITCGQVTSNLAPCLAYLRNTG---PLGRCCGGVKALVNSARTTEDROIACTCLKSAAGAISGINLGKAAGLPSTCGV-NIPYKISPSTDCSKVO $-----M T C G Q V Q G N L A O C I G F L Q K G G V-V P P S-C C T G V K N I L N S S R T T A D R R A V C S C L K A A A G A V R G I N P N N A E A L P G K C G V-N I P Y K I S T S T N C N S I N$ ------ITCGQVSSALAPCIPYVRGGGA-VPPA-CCNGIRNVNNLARTTPDRQAACNCLKOLSASVPGVNPNNAAALPGKCGV-HIPYKISASTNCATVK $-----A I S C G Q V A S A I A P C I S Y A R G Q G S-G P S A G C C S G V R S L N N A A R T T A D R R A A C N C L K N A A A G V S G L N A G N A A S I P S K C G V-S I P Y T I S T S T D C S R V N$

1BWO Type I

1RZL Type I

1MID Type I

1 T12 Type I

1SIY Type I

2ALG Type I

1FK5 Type I

$-------A C Q A S Q L A V--C A S A I L S-G A-K P S G E C C G N L R--------A Q Q G C F C Q Y A K D P T Y G Q Y-I R S P H A R D T L T S C G L-A V P H-------C----$ GVVGVAGAGCNAGQLTV--CTGAIAG-GA-RPTAACCSSLR---------AQQGCFCOFAKDPRYGRY-VNSPNARKAVSSCGI-ALPT-------CH--1TUK Type II ----IDLCGMSDELNECKPAVSKENPTSPSOPCCTALOH-------ADFACLCGYKNSPWLGSFGVDPELASALPKOCGLANAPT------C--1L6H Type II

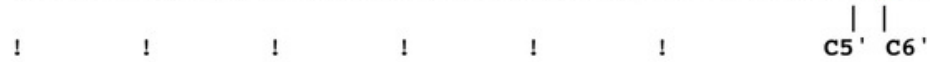
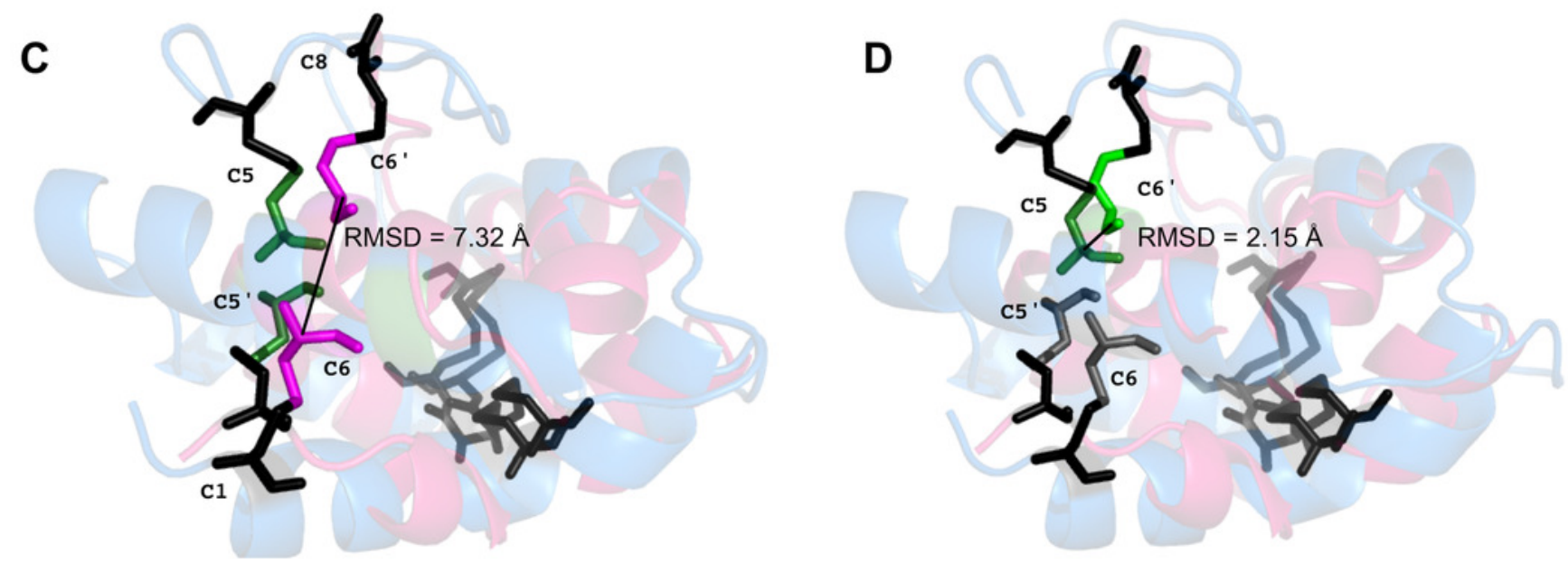


\section{Figure 2}

NsLTP sequence classification.

Dendrogram built on MAFFT alignment of the 797 nsLTP sequences, using Dendroscope program (Huson and Scornavacca 2012). The different nsLTP types are displayed using various colors and the number of sequences in each type is specified in parenthesis. Branch support values of each group are indicated on the corresponding nodes.

Type IV (36)

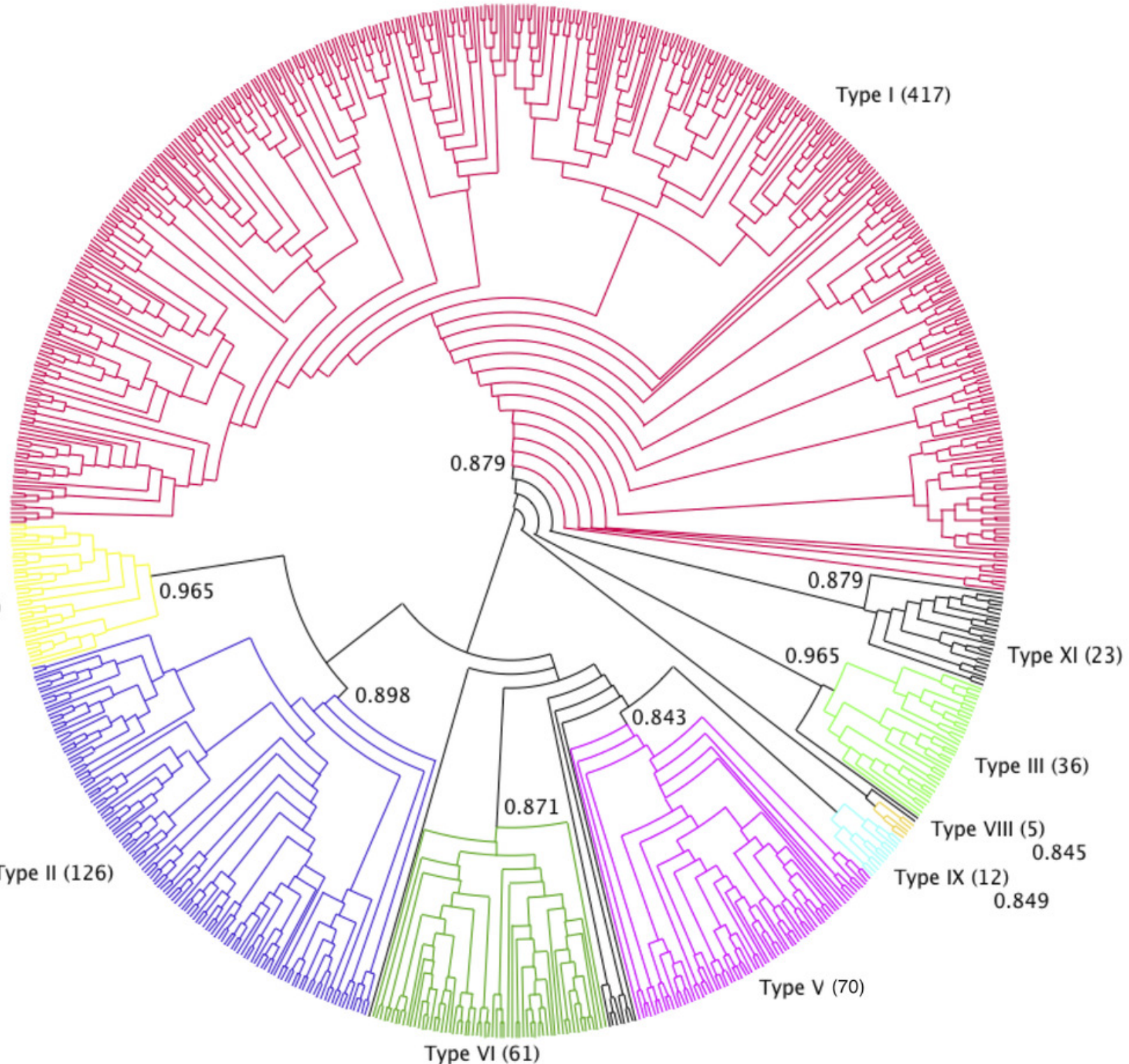




\section{Figure 3}

\section{Consensus sequence alignment for all nsLTP types.}

The indicated amino acids are the most frequent for each type of nsLTP and vertical arrows indicate residues analyzed in detail in the text below.

The residues are colored as follows: $\mathbf{1}$. The sequences were sorted according to the FastME phylogenetic tree order. 2. A color was assigned to each sorted sequence according to a rainbow color gradient order. $\mathbf{3}$. Let $i$ be a position of the alignment, $a(i)$ be an amino acid at position $i$ and $A(a(i), i)$ be the amino acid cluster to which a(i) belongs and which has the lowest Fisher's test probability $F P(A(a(i), i))$ relatively to any tree cluster.

The color of each aligned amino acid a(i) is coded using the Hue-Saturation-Value color space: the color hue of $a$ (i) corresponds to the hue of the median sequence containing an amino acid of the cluster $A(a(i), i)$ at position i. The color saturation of a(i) is proportional to the specificity score $\log (F P(A(a(i), i)))$. The color value or intensity of $a(i)$ is reversely proportional to the conservation score of the column $i$. The more conserved an amino acid cluster is, the darker its color will be, and the more specific to a phylogenetic group an amino acid cluster is, the more saturated its color will be. Consequently, the globally conserved amino acid clusters are highlighted as dark grey or black columns in the sequence alignment while the amino acids cluster specific to a subgroup of related sequences are highlighted by aligned amino acids with same saturated colors. Furthermore, the amino acid specifically conserved in a given protein can then easily be detected by looking at the residues with colors similar to the sequence name color.

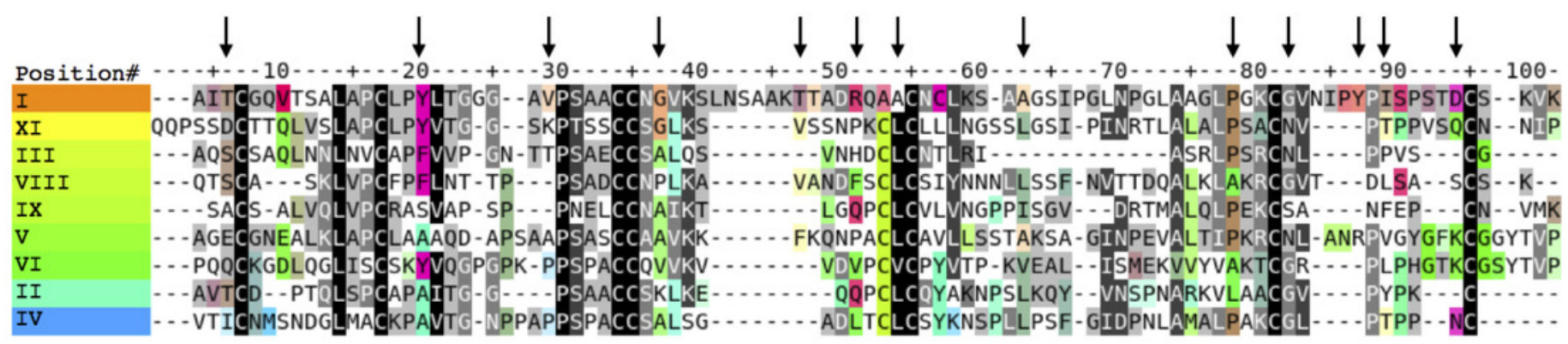


Figure 4

Cartoon representation of the crystallographic structures 1mid (type I), 1tuk (type II) and 2rkn (type IV).

The residues are numbered and colored as in the multiple sequence alignment of Figure 3. The ligands are represented as ball and sticks (carbon in white, oxygen in red). Some determining amino acid side chains are also displayed.
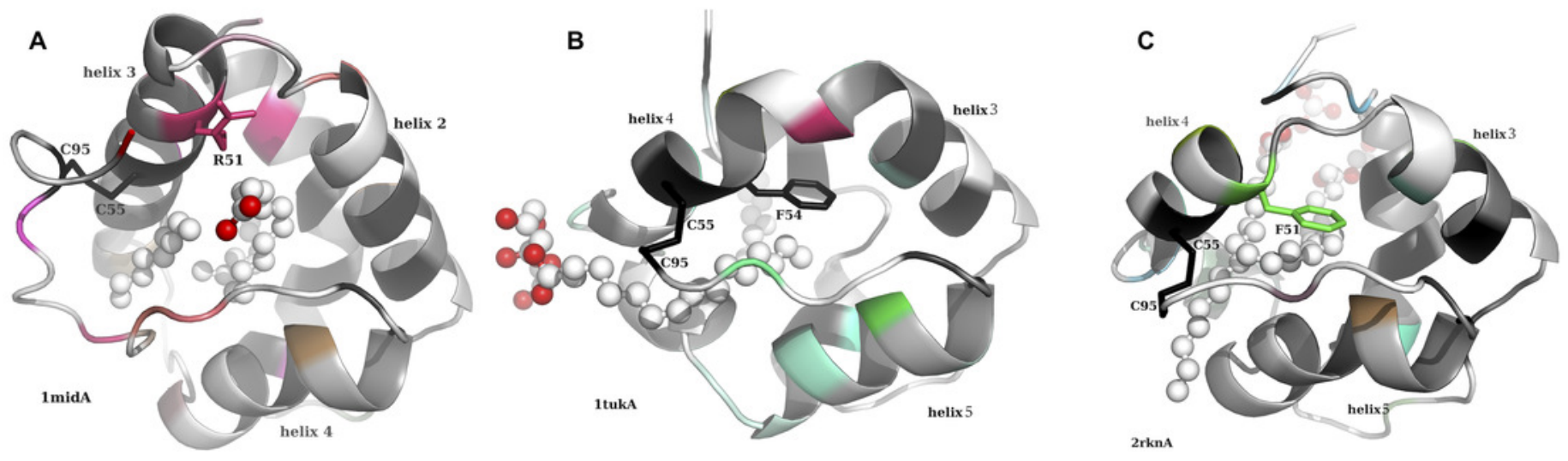


\section{Figure 5}

NsLTP structure classification.

Dendrogram built on Mustang structure-based sequence alignment of the 727 nsLTPs for which a reliable 3D model has been calculated. The two main fold types are displayed in red (type 1 fold) and black (type 2 fold). In order to study their distribution in term of structural families, nsLTP structures are colored according to the previously determined phylogenetic type they belong to (same colors as used in figure 2). Phylogenetic type I nsLTPs display the type 1 fold and all other nsLTPs follow the type 2 fold. 


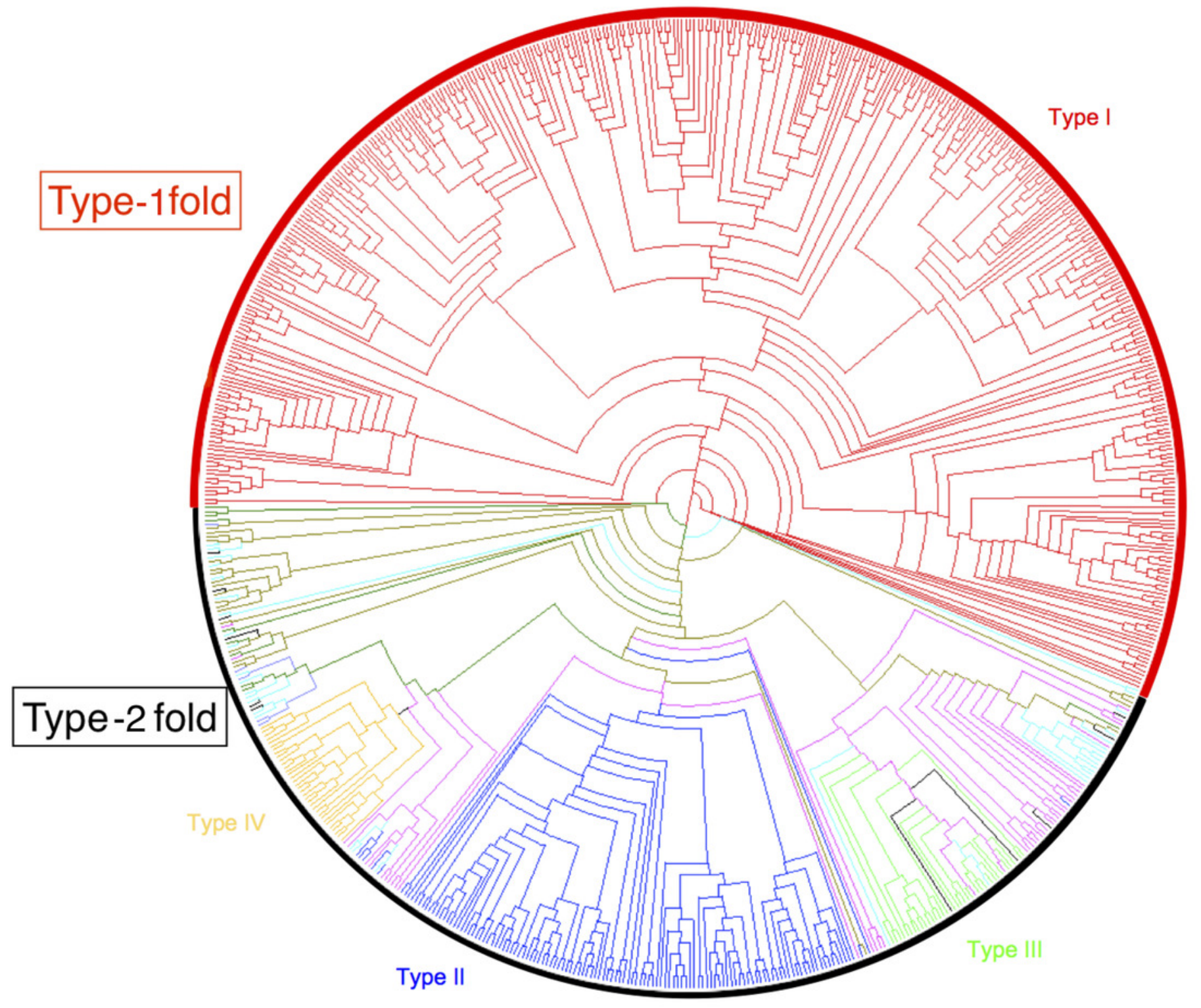




\section{Figure 6}

InTreeGreat view of the structure tree.

The left pane shows the phylogenic tree of the nsLTP structures colored according to type and the right pane represents a close-up of the Type I (colored in red) part of the tree. For clarity, some sub tree parts for which no annotation was available have been collapsed. They appear as grey triangle and the number of structures they contain is indicated. NsLTPs for which a functional annotation is available are highlighted with a grey box in the left column. On the right side of the tree several columns appear that correspond to annotations (PO, $\mathrm{GO}$ ), number of leaves in a collapsed sub-tree together with colored boxes. The first column of boxes shows alternative colors to enhance the clusters, the other ones correspond to each keyword selected among the annotations of the database (here: "defense" or "resistance"). Keywords "defense" or "resistance" used in functional annotation are highlighted with a colored box (blue and red respectively). The "defense cluster" (see text) has been enlarged (black border) for a better view. 


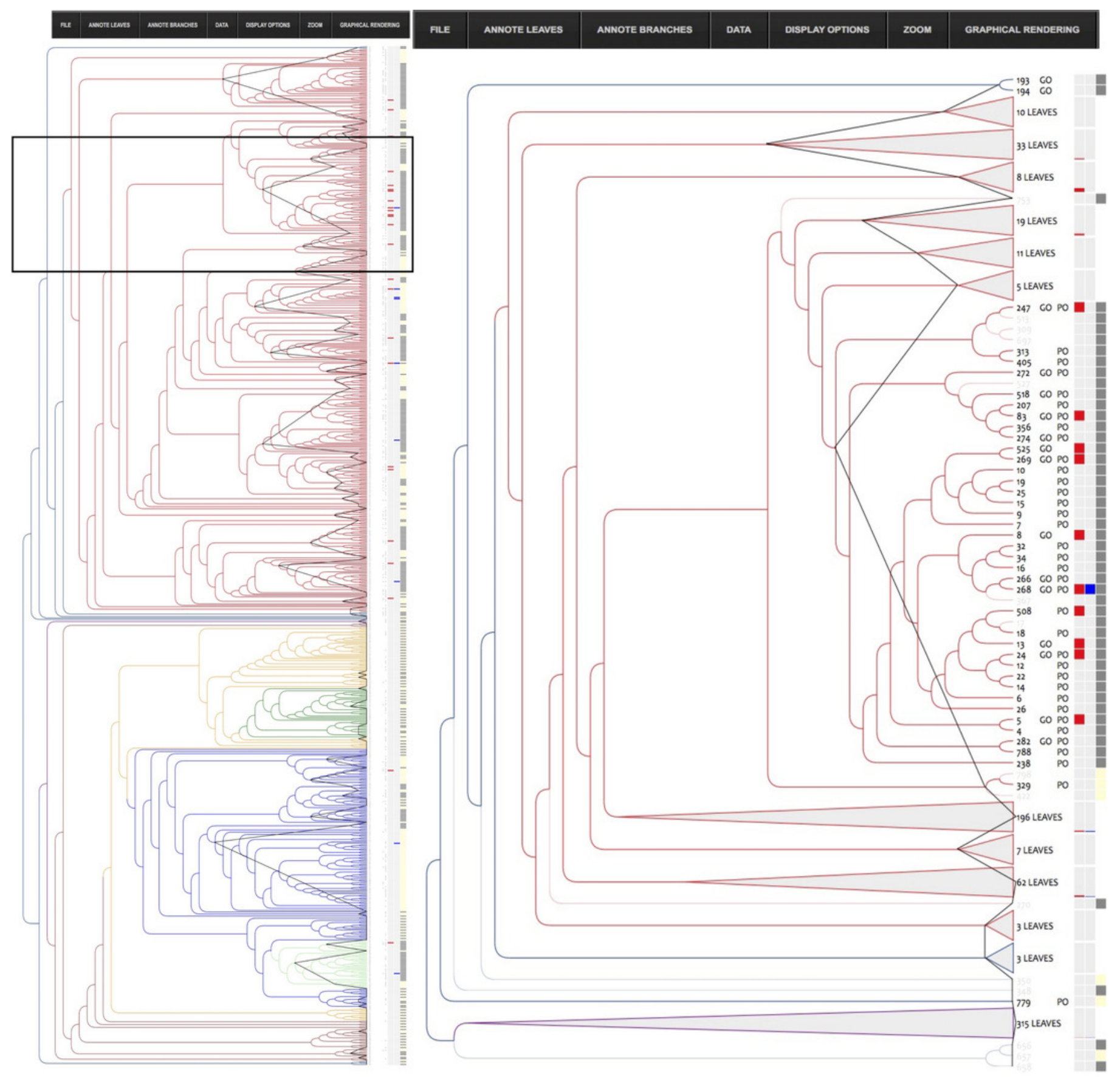




\section{Figure 7}

Conserved amino acid residues among the so-called defense cluster, displayed on the 3D structure of nsLTP 525, (“LTP”, UniProtKB - Q1KMV1).

The more the residue is conserved in the 3D alignment, the redder its color appears, then orange, yellow and green. Residues with no significative conservation appears in white on the figure. Residues highlighted in table 2 and which potential functional implication is discussed (see text) are labeled on the figure. 


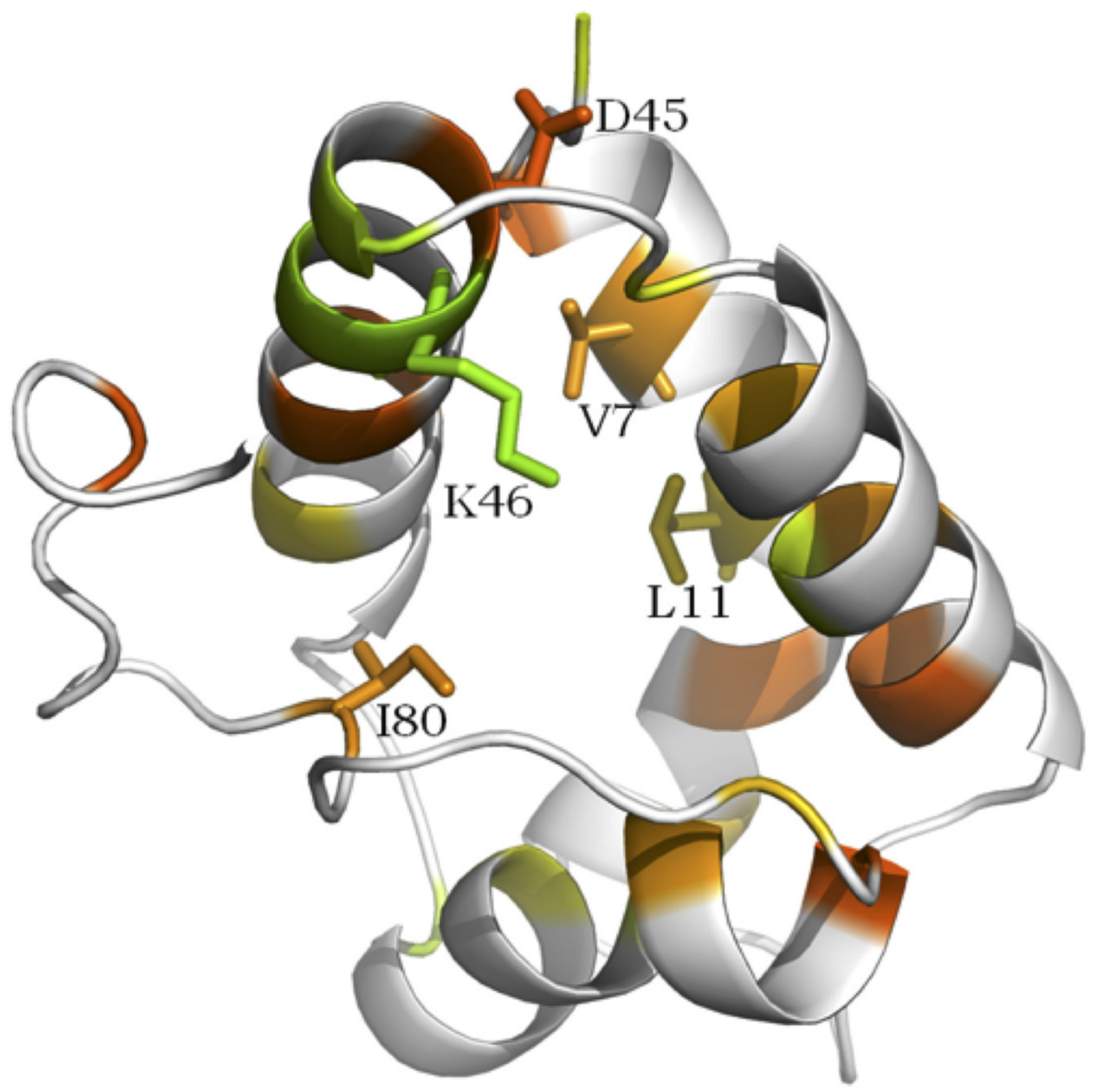




\section{Table $\mathbf{1}$ (on next page)}

Qmean scores obtained by the 797 theoretical models of nsLTPs of this study.

Models obtained by @tome2 present an overall good quality as shown in Table 1 that summarizes the Qmean scores. For $95,85 \%$ of the models, Qmean scores are above 0.4 and $57 \%$ of the models obtained scores ranging from 0.5 to 0.9 , which correspond to scores for high-resolution proteins. It is known that disordered protein regions are very flexible regions. While submitted to automatic evaluation, these flexible regions will be considered as regions of bad quality modeling, leading to lower Qmean scores (Benkert, Tosatto et al. 2008;

Benkert, Biasini et al. 2011). Small proteins tend to have lower scores than larger proteins, because of the lower proportion of secondary structures compared to random coils. However, the set of theoretical models calculated by @tome2 obtained overall good Qmean scores.NB: for 121 theoretical structures, the polypeptide chain could not be fully built and the resulting models were lacking at least one of the 8 cysteine residues. Such models were discarded and a new pool of 677 structures was retained for further analysis. The models are available at: http://atome.cbs.cnrs.fr/AT2B/SERVER/LTP.html 


\begin{tabular}{|c|c|c|}
\hline Qmean score (Q) & Nb. of models & Dataset proportion \\
\hline$Q<0.2$ & 0 & $0 \%$ \\
\hline $0.2<Q<0.3$ & 1 & $0.1 \%$ \\
\hline $0.3<Q<0.4$ & 30 & $4 \%$ \\
\hline $0.4<Q<0.5$ & 173 & $22 \%$ \\
\hline $0.5<Q<0.6$ & 309 & $39 \%$ \\
\hline $0.6<Q<0.7$ & 221 & $28 \%$ \\
\hline $0.7<Q<0.8$ & 43 & $5 \%$ \\
\hline $0.8<Q<0.9$ & 9 & $1 \%$ \\
\hline $0.9<Q$ & 1 & $0.1 \%$ \\
\hline
\end{tabular}

1

2

3

4 


\section{Table 2 (on next page)}

Compared analysis of Evolutionary Trace of three groups of nsLTPs.

Compared analysis of Evolutionary Trace of three groups of nsLTPs: (A) the defense cluster (43 proteins), (B) the cluster containing all type 1 fold nsLTPs (402 proteins) and (C) a group composed by all type 1 fold defense/resistance nsLTPs, including those which do not belong to the defense cluster (28 proteins). This table lists the 30\% top-ranked (= most conserved) residues identified in the defense cluster trace and shows, by comparison, the ranking of these same residues in the other two traces, together with their coverage, variability, and rvET score. Residue positions in the reference proteins and in the structure-based sequence alignment are also indicated. Alignment position is the same in all three groups because all three alignments used to perform the traces are extracted from the general multiple alignments of all 797 nsLTPs of the study. Five residues are highlighted for they are differently conserved in the three clusters of proteins (see text). 


\begin{tabular}{|c|c|c|c|c|c|c|}
\hline \multicolumn{7}{|l|}{ A } \\
\hline \multicolumn{7}{|c|}{ Defense cluster (ref. prot. $=525$ ) } \\
\hline Rank & $\begin{array}{l}\text { Residue } \\
\text { Number }\end{array}$ & $\begin{array}{l}\text { Alignment } \\
\text { Position }\end{array}$ & Residue & Coverage & Variability & $\begin{array}{l}\text { rvET } \\
\text { score }\end{array}$ \\
\hline 1 & 4 & 93 & C & 0.10000 & $\mathrm{C}$ & 1.00 \\
\hline 1 & 14 & 159 & C & 0.10000 & C & 1.00 \\
\hline 1 & 29 & 228 & C & 0.10000 & C & 1.00 \\
\hline 1 & 30 & 229 & C & 0.10000 & C & 1.00 \\
\hline 1 & 45 & 259 & D & 0.10000 & D & 1.00 \\
\hline 1 & 50 & 275 & $\mathrm{C}$ & 0.10000 & $\mathrm{C}$ & 1.00 \\
\hline 1 & 52 & 277 & $\mathrm{C}$ & 0.10000 & C & 1.00 \\
\hline 1 & 72 & 372 & C & 0.10000 & C & 1.00 \\
\hline 1 & 86 & 432 & C & 0.10000 & C & 1.00 \\
\hline 10 & 7 & 137 & V & 0.13333 & AV & 1.11 \\
\hline 11 & 32 & 231 & G & 0.13333 & SG & 1.11 \\
\hline 12 & 80 & 402 & I & 0.13333 & VI & 1.11 \\
\hline 13 & 69 & 367 & $P$ & 0.14444 & PA & 1.17 \\
\hline 14 & 36 & 236 & $\mathrm{~L}$ & 0.15556 & LV & 1.28 \\
\hline 15 & 17 & 165 & $Y$ & 0.16667 & FY & 1.59 \\
\hline 16 & 74 & 374 & V & 0.17778 & LVIA & 1.75 \\
\hline 17 & 11 & 154 & $\mathbf{L}$ & 0.18889 & LV & 1.83 \\
\hline 18 & 54 & 289 & K & 0.20000 & VKQ & 1.93 \\
\hline 19 & 65 & 360 & A & 0.21111 & TALV & 2.01 \\
\hline 20 & 40 & 247 & $A$ & 0.22222 & TAV & 2.13 \\
\hline 21 & 1 & 63 & A & 0.23333 & AD & 2.15 \\
\hline 22 & 33 & 232 & V & 0.24444 & AVI & 2.29 \\
\hline 23 & 68 & 364 & I & 0.25556 & LI & 2.50 \\
\hline 24 & 43 & 256 & $\mathrm{~T}$ & 0.26667 & TPMAS & 2.61 \\
\hline 25 & 61 & 344 & $\mathrm{~N}$ & 0.27778 & KNSV & 2.65 \\
\hline 26 & 47 & 268 & $Q$ & 0.28889 & RQK & 2.71 \\
\hline 27 & 46 & 266 & K & 0.30000 & RK & 2.75 \\
\hline \multicolumn{7}{|l|}{ B } \\
\hline \multicolumn{7}{|c|}{ Fold 1 nsLTPs (ref. prot. = 437) } \\
\hline Rank & $\begin{array}{l}\text { Residue } \\
\text { Number }\end{array}$ & $\begin{array}{c}\text { Alignment } \\
\text { Position }\end{array}$ & Residue & Coverage & Variability & $\begin{array}{l}\text { rvET } \\
\text { score }\end{array}$ \\
\hline 1 & 14 & 159 & $\mathrm{C}$ & 0.05376 & $\mathrm{C}$ & 1.00 \\
\hline 1 & 29 & 228 & C & 0.05376 & C & 1.00 \\
\hline 1 & 30 & 229 & C & 0.05376 & C & 1.00 \\
\hline 1 & 50 & 275 & C & 0.05376 & C & 1.00 \\
\hline 1 & 52 & 277 & $\mathrm{C}$ & 0.05376 & C & 1.00 \\
\hline 6 & 75 & 372 & C & 0.06452 & CR & 1.75 \\
\hline 7 & 4 & 93 & C & 0.07527 & CA & 3.00 \\
\hline 8 & 89 & 432 & C & 0.08602 & CDN & 4.36 \\
\hline 9 & 72 & 367 & $\mathrm{P}$ & 0.09677 & PASLQG & 7.27 \\
\hline 10 & 46 & 266 & $\mathbf{R}$ & 0.10753 & RKTAPIQD & 11.55 \\
\hline 11 & 7 & 137 & V & 0.11828 & VALISGT & 11.81 \\
\hline 12 & 32 & 231 & G & 0.12903 & GSAEQVHR & 13.26 \\
\hline
\end{tabular}




\begin{tabular}{|c|c|c|c|c|c|c|}
\hline 13 & 36 & 236 & L & 0.13978 & LVIM & 13.58 \\
\hline 14 & 77 & 374 & V & 0.15054 & VLTAINP & 13.66 \\
\hline 15 & 17 & 165 & $Y$ & 0.16129 & YFAH & 13.82 \\
\hline 16 & 40 & 247 & $A$ & 0.17204 & ATSVIRPL & 14.49 \\
\hline 17 & 68 & 360 & $A$ & 0.18280 & ATVLFIM & 14.52 \\
\hline 18 & 71 & 364 & I & 0.19355 & LIVTAPFM & 14.53 \\
\hline 19 & 54 & 289 & $\mathrm{~K}$ & 0.20430 & KVQIERLMHTS & 15.40 \\
\hline 20 & 45 & 259 & $\mathbf{D}$ & 0.21505 & DAENITLRG.K & 15.74 \\
\hline 21 & 83 & 402 & I & 0.22581 & IVFPLTAKW & 15.92 \\
\hline 29 & 33 & 232 & V & 0.31183 & VAILSM & 21.38 \\
\hline 32 & 47 & 268 & $\mathrm{R}$ & 0.34409 & KQRVEMIYSH & 24.45 \\
\hline 34 & 11 & 154 & I & 0.36559 & VLMIFATP & 25.38 \\
\hline 42 & 64 & 344 & $\mathrm{~N}$ & 0.45161 & NGKQDASTLERVFYI & 54.16 \\
\hline 56 & 43 & 256 & $\mathrm{~T}$ & 0.60215 & TAPGRSQKDHVMI.LFY & 38.13 \\
\hline 61 & 1 & 63 & A & 0.65591 & AHETDVPSGFQL & 39.96 \\
\hline \multicolumn{7}{|l|}{ C } \\
\hline \multicolumn{7}{|c|}{ Defense nsLTPs outside cluster (ref. prot. $=525$ ) } \\
\hline Rank & $\begin{array}{l}\text { Residue } \\
\text { Number }\end{array}$ & $\begin{array}{l}\text { Alignment } \\
\text { Position }\end{array}$ & Residue & Coverage & Variability & $\begin{array}{l}\text { rvET } \\
\text { score }\end{array}$ \\
\hline 1 & 4 & 93 & $\mathrm{C}$ & 0.11111 & C & 1.00 \\
\hline 1 & 14 & 159 & C & 0.11111 & C & 1.00 \\
\hline 1 & 29 & 228 & C & 0.11111 & C & 1.00 \\
\hline 1 & 30 & 229 & C & 0.11111 & C & 1.00 \\
\hline 1 & 50 & 275 & C & 0.11111 & C & 1.00 \\
\hline 1 & 52 & 277 & C & 0.11111 & C & 1.00 \\
\hline 1 & 72 & 372 & C & 0.11111 & C & 1.00 \\
\hline 1 & 86 & 432 & C & 0.11111 & C & 1.00 \\
\hline 1 & 7 & 137 & V & 0.11111 & V & 1.00 \\
\hline 1 & 69 & 367 & $P$ & 0.11111 & $\mathrm{P}$ & 1.00 \\
\hline 11 & 45 & 259 & D & 0.13333 & DL & 1.15 \\
\hline 12 & 80 & 402 & $\mathrm{I}$ & 0.13333 & IW & 1.15 \\
\hline 13 & 74 & 374 & $\mathrm{~V}$ & 0.15556 & VIN & 1.39 \\
\hline 16 & 17 & 165 & Y & 0.18889 & YF & 1.67 \\
\hline 17 & 36 & 236 & L & 0.18889 & LI & 1.67 \\
\hline 18 & 32 & 231 & G & 0.20000 & GAV & 1.76 \\
\hline 20 & 54 & 289 & $\mathrm{~K}$ & 0.22222 & KVQ & 1.93 \\
\hline 22 & 65 & 360 & A & 0.24444 & AVF & 2.04 \\
\hline 23 & 40 & 247 & A & 0.25556 & ATVS & 2.05 \\
\hline 25 & 33 & 232 & V & 0.27778 & VALI & 2.59 \\
\hline 27 & 61 & 344 & $N$ & 0.30000 & NVDR & 2.88 \\
\hline 30 & 46 & 266 & K & 0.33333 & KR & 3.25 \\
\hline 31 & 11 & 154 & $\mathbf{L}$ & 0.34444 & LIVM & 3.26 \\
\hline 36 & 43 & 256 & $\mathrm{~T}$ & 0.40000 & TPQRS & 3.72 \\
\hline 38 & 68 & 364 & 1 & 0.42222 & ILV & 3.95 \\
\hline 44 & 47 & 268 & Q & 0.48889 & QRK & 4.61 \\
\hline 45 & 1 & 63 & A & 0.50000 & A.QV & 4.63 \\
\hline
\end{tabular}

